Pacific Journal of Mathematics

REPRESENTATIONS AND AUTOMORPHISMS OF THE
IRRATIONAL ROTATION ALGEBRA 


\title{
REPRESENTATIONS AND AUTOMORPHISMS OF THE IRRATIONAL ROTATION ALGEBRA
}

\author{
BERNDT A. BRENKEN
}

\begin{abstract}
Given an irrational number $\alpha, A_{\alpha}$ is the unique $C^{*}$-algebra generated by two unitary operators, $U$ and $V$, satisfying the twisted commutation relation $U V=\exp (2 \pi i \alpha) V U$. We investigate separable representations of $A_{\alpha}$ which, when restricted to the abelian $C^{*}$ algebra generated by $V$, are of uniform multiplicity $m$. These representations are classified by their multiplicity, a quasi-invariant Borel measure on the circle (w.r.t. rotation by the angle $2 \pi \alpha$ ) and a unitary one cocycle.

Separable factor representations lie in this class, the measure being ergodic in this case. A factor representation is of uniform multiplicity $m^{\prime}$ on the $C^{*}$ algebra generated by $U$, and if $m, m^{\prime}$ are relatively prime, the representation is irreducible. By use of an action of $\operatorname{SL}(2, \mathbf{Z})$ as *-automorphisms of $A_{\alpha}$, that we construct, we arrive at a separating family of pure states of $A_{\alpha}$ whose corresponding irreducible representations provide explicit examples with $m$ and $m^{\prime}$ occurring as any given pair of nonzero relatively prime numbers.
\end{abstract}

Introduction. We study representations of the irrational rotation algebras, a special class of $C^{*}$-algebras that has received a great deal of attention in recent years [13-16]. Our focus is primarily, though not exclusively, on factor and, in particular, irreducible, representations of algebras in this family. This class of algebras is parametrized by the irrational numbers in $[0,1]$. To each irrational number $\alpha$ in $[0,1]$, we make correspond the $C^{*}$-algebra $A_{\alpha}$ generated by multiplications by continuous functions on $\mathbf{T}$, the unit circle in the plane of complex numbers, and the unitary transformation on $L_{2}(\mathbf{T}, \nu)$ arising from rotation of $\mathbf{T}$ through the angle $2 \pi \alpha$, where $\nu$ is (normalized) Haar measure on $\mathbf{T}$. More specifically, let $M_{f} g$ be $f g$ where $f \in C(\mathbf{T})$ and $g \in L_{2}(\mathbf{T}, \nu)$, and let $(U g)(\exp (2 \pi i \theta))$ be $g(\exp (2 \pi i(\theta+\alpha)))$ for each $\theta$ in $[0,1]$. Then $A_{\alpha}$ is the $C^{*}$-algebra generated by $\left\{M_{f}, U: f \in C(\mathbf{T})\right\}$.

Although we have described $A_{\alpha}$ in a particular representation, in the first instance, it can be characterized (uniquely, as it turns out) as a $C^{*}$-algebra generated by two unitary operators $U$ and $V$ satisfying a "twisted" commutation relation $U V=(\exp 2 \pi i \alpha) V U$. In the representation of $A_{\alpha}$ we described, $U$ is as noted and $V$ is multiplication by $z$ (the identity transform on $\mathbf{T}$ ). There are several other ways of viewing $A_{\alpha}$ that will be useful to us. The rotation of $\mathbf{T}$ through the angle $2 \pi \alpha$ is a 
homeomorphism that engenders an automorphism of $C(\mathbf{T})$. We use ' $\alpha$ ' to denote this automorphism as well as the parametrizing irrational number. The mapping that assigns $\alpha^{n}$ to the integer $n$ is a homomorphism of $\mathbf{Z}$, the additive group of integers, into Aut $C(\mathbf{T})$, the group of automorphisms of (the abelian $C^{*}$-algebra) $C(\mathbf{T})$. Such a homomorphism is termed an "action" of $\mathbf{Z}$ on $C(\mathbf{T})$ and the triple $(C(\mathbf{T}), \alpha, \mathbf{Z})$ is a $C^{*}$-dynamical system. A general construction associates a $C^{*}$-algebra with such a dynamical system, the crossed product, $C(\mathbf{T}) \times_{\alpha} \mathbf{Z}$, of $C(\mathbf{T})$ by $\mathbf{Z}$ via the action. In the present case the crossed product is another description of $A_{\alpha}$.

From [5] the representations of $A_{\alpha}$ are in one-to-one (canonical) correspondence with the "covariant representations" of the $C^{*}$-dynamical system $(C(\mathbf{T}), \alpha, \mathbf{Z})$. That is, a representation $\tilde{\pi}$ of $A_{\alpha}$ corresponds to a representation $\pi$ of $C(\mathbf{T})$ and a unitary representation of $\mathbf{Z}$ such that the action of $\mathbf{Z}$ on $C(\mathbf{T})$ is implemented by the unitary operators representing $\mathbf{Z}$ on $\pi(C(\mathbf{T}))$. In our situation $C(\mathbf{T})$ "appears" as a subalgebra of $A_{\alpha}$, and $\mathbf{Z}$ "appears" as a group of unitary elements in $A_{\alpha}$ (and together they generate $A_{\alpha}$ ) - much as in our initial (representation) description of $A_{\alpha}$. Given $\tilde{\pi}$, then, $\pi$ is the restriction of $\tilde{\pi}$ to $C(\mathbf{T})$ and the unitary representation of $\mathbf{Z}$ is the restriction of $\tilde{\pi}$ to the unitary elements representing $\mathbf{Z}$ in $A_{\alpha}$. We make use of the well-developed representation theory of abelian $C^{*}$-algebras to single out those representations $\tilde{\pi}$ of $A_{\alpha}$ whose associated $\pi$ has "uniform multiplicity". That is, we study those $\tilde{\pi}$ for which $\pi(C(\mathbf{T}))$ acts as an $m$-fold copy of a representation of $C(\mathbf{T})$ in which the strong-operator closure of the image is maximal abelian - in another form, the commutant $\pi(C(\mathbf{T}))^{\prime}$ of $\pi(C(\mathbf{T}))$ is a von Neumann algebra of type $I_{m}$. Here, $m$ is a given cardinal number and the representation $\tilde{\pi}$ is said to be a "uniform multiplicity $m$ representation" (of $A_{\alpha}$ ). We will be concerned exclusively with the case of separable Hilbert space representations, so that $m$ is either finite or $\boldsymbol{\aleph}_{0}$.

To each (separable) uniform multiplicity representation $\tilde{\pi}$ of $A_{\alpha}$, we associate a triple $(m, \nu, b)$, where $m$ is the multiplicity of $\pi, \nu$ is a quasi-invariant regular Borel measure on $\mathbf{T}$, and $b$ is a certain "unitary cocycle" (on $\mathbf{Z}$ with values in the unitary group of the algebra of $m \times m$ matrices with entries in $\left.L^{\infty}(\mathbf{T}, \nu)\right)$. We prove that each factor representation of $A_{\alpha}$ is a uniform multiplicity representation, and $\nu$ is ergodic under the action of $\alpha$, in this case. Two uniform multiplicity representations of $A_{\alpha}$ are unitarily equivalent if and only if they have "equivalent" triples: the multiplicities are the same, the measures on $\mathbf{T}$ are equivalent (absolutely continuous with respect to one another), and the unitary cocycles 
are cohomologous. We establish corresponding criteria for quasi-equivalence of uniform multiplicity representations. In case $m$ is finite we show that the representation $\tilde{\pi}$ is of type I (that is, $\tilde{\pi}\left(A_{\alpha}\right)^{\prime \prime}$ is a von Neumann algebra of type I).

Of course $A_{\alpha}$ has pure states and each of these gives rise to an irreducible representation (by means of the GNS construction). Each irreducible representation is a factor representation (of type $I_{\infty}$ ) and is, accordingly, a uniform multiplicity representation. By exploiting the symmetry of the two generating unitary elements $U$ and $V$ (the abelian $C^{*}$-algebra generated by $U$ is also isomorphic to $C(\mathbf{T})$ ), we may associate with each factor representation of $A_{\alpha}$ a second cardinal number $m^{\prime}$ (determined by the multiplicity of the restriction of the representation to the subalgebra generated by $U$ ). We prove that the representation is irreducible when $m$ and $m^{\prime}$ are relatively prime.

It is clear that there are multiplicity one representations (that is, ones for which $\pi(C(T))^{\prime \prime}$ is maximal abelian). These abound - indeed, the representation in which we first described $A_{\alpha}$ is such. What is not clear $a$ priori is that there are irreducible representations of multiplicity greater than one [1]. In $\$ 2.5$ we construct such a representation for each finite multiplicity $m$, where $\nu$ is Haar measure. In effect we have found explicit ergodic actions of $\mathbf{Z}$ on the algebra of $m \times m$ matrices with entries in $L^{\infty}(\mathbf{T}, \nu)$. In fact we find irreducible representations of $A_{\alpha}$ such that the pair of multiplicities $m$ and $m^{\prime}$ obtained by restricting the representation to the $C^{*}$-algebras generated by $V$ and $U$ respectively are any given pair of relatively prime numbers. This is accomplished with the aid of an action of $\operatorname{SL}(2, \mathbf{Z})$ on $A_{\alpha}$ that we construct. Loosely speaking, we let an element (matrix) of $\operatorname{SL}(2, \mathbf{Z})$ act on the "vector" $(p, q)$ of integers appearing in $V^{p} U^{q}$ and modify this by a (carefully chosen) phase factor to determine the automorphism of $A_{\alpha}$ corresponding to that element of $\operatorname{SL}(2, \mathbf{Z})$. With $\theta$ in $[0,1]$, "evaluation" at $\exp 2 \pi i \theta$ is a pure state of $C(\mathbf{T})$ and has a pure state extension $\varphi_{\theta}$ to $A_{\alpha}$. For each $g$ in $\operatorname{SL}(2, \mathbf{Z}), \varphi_{\theta} \circ \beta_{g}$ is a pure state of $A_{\alpha}$, where $\beta_{g}$ is the automorphism of $A_{\alpha}$ corresponding to $g$. With $g$ suitably selected, the GNS representation of $A_{\alpha}$ corresponding to $\varphi_{\theta} \cdot \beta_{g}$ has the given multiplicities $m$ and $m^{\prime}$.

We show that the pure states $\varphi_{\theta}$ and $\varphi_{\tilde{\theta}}$ of $A_{\alpha}$ are unitarily equivalent if and only if $\exp 2 \pi i \theta$ lies in the orbit of $\exp 2 \pi i \tilde{\theta}$ under the action of $\alpha$ (on T). We determine when $\varphi_{\theta}$ and $\varphi_{\tilde{\theta}}^{\circ} \beta_{g}$ are unitarily equivalent. If $H$ is the subgroup of $\operatorname{SL}(2, \mathbf{Z})$ consisting of matrices of the form $\left[\begin{array}{ll}1 & n \\ 0 & 1\end{array}\right](n \in \mathbf{Z})$ and $g \in H$, we show that $\varphi_{\theta} \circ \beta_{g}=\varphi_{\theta}$ and $\varphi_{\theta} \circ \beta_{-g}=\varphi_{1-\theta}$. We prove that if $\varphi_{\theta}^{\circ} \circ \beta_{g}$ and $\varphi_{\theta}$ are unitarily equivalent, then $g$ or $-g$ is in $H$, whence 
$\varphi_{\tilde{\theta}} \circ \beta_{g}$ and $\varphi_{\theta}$ are unitarily equivalent if and only if either $g \in H$ and $\exp 2 \pi i \tilde{\theta}$ is in the orbit of $\exp 2 \pi i \theta$ or $-g \in H$ and $\exp -2 \pi i \tilde{\theta}$ is in the orbit of $\exp 2 \pi i \theta$. It is also proved that the pure states of the form $\varphi_{\theta} \circ \beta_{g}$ "separate" $A_{\alpha}$.

Explicit computation of the unitary cocycle is made in several cases, in particular, for the "multiplicity $m$ " irreducible representations described above. We define certain cocycles to be "diagonal" and compute for uniform multiplicity $m$ representations ( $m \in\left\{1,2, \ldots, \boldsymbol{\aleph}_{0}\right\}$ ) with diagonal cocycles the structure of the commutant of the image of the representation. The particular cocycle (its diagonal entries) imposes an equivalence relation on the set of pairs of integers $1, \ldots, m$. This relation consists of all pairs if and only if the representation is factorial. If the relation coincides with "equality", then the commutant is abelian. When a uniform multiplicity representation has multiplicity $\boldsymbol{}_{0}$ and the cocycle arises from the "bilateral shift" the representation is the familiar "group-measure space construction" of Murray and von Neumann [17]. It is of type $\mathrm{II}_{1}$ and arises from the trace on $A_{\alpha}$. In this connection, we make use of several basic algebraic facts about $A_{\alpha}$ (see, for example, $[12,20]$ ). It possesses a unique tracial state, whence all finite representations of $A_{\alpha}$ are type $\mathrm{II}_{1}$ factor representations and each is unitarily equivalent to the trace representation if it is cyclic [8]. In addition, $A_{\alpha}$ is simple, and, as noted earlier, it is characterized as being generated by two unitary elements $U$ and $V$ satisfying the twisted commutation relation $U V=(\exp 2 \pi i \alpha) V U$.

The recently developed $K$-theory of $C^{*}$-algebras (see, for example, [13]) supplies us with a natural map of Aut $A_{\alpha}$ to the group of automorphisms of $\mathbf{Z} \times \mathbf{Z}\left(=K_{1}\left(A_{\alpha}\right)\right)$. This map is the identity on $\operatorname{SL}(2, \mathbf{Z})$ and we show that the kernel of this map (a closed subgroup of Aut $A_{\alpha}$ in an appropriate topology) contains the centrally trivial automorphisms (see [3, 7]) and, thus, also the approximately inner automorphisms of $A_{\alpha}$.

There are many questions related to the representation theory of $A_{\alpha}$ on which we have not touched (the conjugacy of the maximal abelian subalgebras of $A_{\alpha}$ and criteria for type III representations, for example) and some for which our information is not complete (a computable necessary and sufficient condition for factoriality of a representation, for example). We expect to return to these questions in later publications.

During the course of these investigations, we have benefited from conversations with several of our colleagues at the University of Pennsylvania. It is a pleasure to express our gratitude to Joachim Cuntz, Vaughn Jones, Robert Powers, Jonathan Rosenberg, and Antony Wassermann. A special debt of gratitude is due to $\mathbf{R}$. Kadison who suggested the general 
topic and whose patient and careful supervision of my thesis, from which this paper is adapted, has led to several simplifications and improvements.

1. The irrational rotation algebra. A $C^{*}$-dynamical system is a triple ( $A, \alpha, G$ ) where $A$ is a $C^{*}$-algebra, $G$ a locally compact group and $\alpha$ a homomorphism of $G$ into the group of *-automorphisms of $A$ such that $x \rightarrow \alpha_{x}(b)$ is continuous from $G$ to $A$ for each $b \in A$. Given a $C^{*}$-dynamical system we may form a Banach-*-algebra $L^{1}(G, A)$. If $C_{c}(G, A)$ is the vector space of continuous functions with compact supports from $G$ to $A$ we endow it with a norm, involution and "twisted" convolution product as follows $[5,12,20]$

$$
\begin{gathered}
\|f\|_{1}=\int|f(x)| d x, \\
f^{*}(x)=\Delta(x)^{-1} \alpha_{x}\left(f\left(x^{-1}\right)\right)^{*}, \\
(f * h)(x)=\int_{G} f(s) \alpha_{s}\left(h\left(s^{-1} x\right)\right) d s \quad\left(f, h \in C_{c}(G, A), x \in G\right) .
\end{gathered}
$$

Here $d g$ and $\Delta(g)$ denote the left Haar measure and the modular function on $G$ respectively. With this structure $C_{c}(G, A)$ is a normed *-algebra with isometric involution and $L^{1}(G, A)$ denotes its completion. A covariant representation of a $C^{*}$-dynamical system $(A, \alpha, G)$ is a triple $(\pi, U, H)$ where $\pi$ is a representation of $A$ as bounded operators on the Hilbert space $H, U$ is a strongly continuous unitary representation of $G$ on $H$, and $U_{x} \pi(b) U_{x}^{*}=\pi\left(\alpha_{x}(b)\right)$ for $x$ in $G, b$ in $A$.

Proposition 1.1 [5]. If $(\pi, U, H)$ is a covariant representation of the $C^{*}$-dynamical system $(A, \alpha, G)$, there is a nondegenerate representation $(\pi \times U, H)$ of $L^{1}(G, A)$ such that

$$
(\pi \times U)(f)=\int_{G} \pi(f(x)) U_{x} d x \text { for } f \text { in } C_{c}(G, A) .
$$

The correspondence $(\pi, U, H) \rightarrow(\pi \times U, H)$ is a bijection onto the set of nondegenerate representations of $L^{1}(G, A)$.

The crossed product associated with the system $(A, \alpha, G)$, denoted by ' $A \times{ }_{\alpha} G$ ', is the enveloping $C^{*}$-algebra of $L^{1}(G, A)$. The following " universal" property for $A \times_{\alpha} G$ is a consequence of the preceding proposition and the universal property of enveloping $C^{*}$-algebras [4]. 
Proposition 1.2. Let $\tau$ be the canonical map of $L^{1}(G, A)$ into $A \times{ }_{\alpha} G$. If $(\pi, U, H)$ is a covariant representation of $(A, \alpha, G)$, there is a unique representation ( $\tilde{\pi}, H)$ of $A \times G$ such that the representation $\tilde{\pi} \circ \tau$ of $L^{1}(G, A)$ is $\pi \times U$.

Notice that if the group $G$ is discrete and $A$ is a $C^{*}$-algebra with unit, then $G$ and $A$ have canonical isomorphic copies in $A \times{ }_{\alpha} G$. For if $a \in A$, the function from $G$ to $A$ defined at $e$ (the unit of $G$ ) by $a$, and taking the value 0 elsewhere, is an element of $C_{c}(G, A)\left(\subseteq L^{1}(G, A)\right)$ representing $a$. We represent $x$ in $G$ as the element of $C_{c}(G, A)$ that maps $x$ to $\operatorname{Id}_{A}$ and has the value 0 elsewhere on $G$. With these identifications the unit of $A$ is a unit for $A \times_{\alpha} G$.

We now define the irrational rotation algebra $A_{\alpha}$. If $\alpha$ is an irrational number in $[0,1]$ define an action of the discrete abelian group $\mathbf{Z}$ via homeomorphisms of the circle group $\mathbf{T}$ by $n: e^{2 \pi i t} \rightarrow e^{2 \pi i(t+n \alpha)}$. This results in an action $\alpha$ of $\mathbf{Z}$ on Borel functions $f$ on $\mathbf{T}$ given by $\left(\alpha_{n} f\right)\left(e^{2 \pi t t}\right)$ $=f\left(e^{2 \pi i(t+n \alpha)}\right)$. This action is by ${ }^{*}$-automorphisms on the abelian $C^{*}$-algebra $C(\mathbf{T})$. Define $A_{\alpha}$ to be the crossed product $C(T) \times{ }_{\alpha} \mathbf{Z}$ of the $C^{*}$-dynamical system $(C(\mathbf{T}), \alpha, \mathbf{Z})$. As $\mathbf{Z}$ is discrete and $C(\mathbf{T})$ has a unit, $\mathbf{Z}$ and $C(\mathbf{T})$ have canonical 'copies' in $C(\mathbf{T}) \times{ }_{\alpha} \mathbf{Z}$. Denote by $U_{n}$ the unitary operator in $C(\mathbf{T}) \times{ }_{\alpha} \mathbf{Z}$ corresponding to the element $n$ of $\mathbf{Z}$. For $f \in$ $C_{c}(\mathbf{Z}, C(\mathbf{T})), f=\sum f(n) U_{n}$ (where the product $f(n) U_{n}$ is the "twisted" convolution product).

The one-to-one correspondence between representations $\pi$ of $A_{\alpha}$ and covariant representations $(\rho, T)$ of $(C(\mathbf{T}), \alpha, Z)$ is given by restriction, i.e. $\left.\pi\right|_{C(\mathbf{T})}=\rho$ and $\pi\left(U_{n}\right)=T(n)(n \in \mathbf{Z})$. There is an action $t \rightarrow \hat{\alpha}_{t}$ of $\mathbf{T}$ $(=\hat{\mathbf{Z}})$ on $A_{\alpha}$, the dual action, given by

$$
\hat{\alpha}_{t}(f)(n)=t^{n} f(n) \quad\left(f \in C_{c}(\mathbf{Z}, C(\mathbf{T})), t \in \mathbf{T}, n \in \mathbf{Z}\right)
$$

We may define a conditional expectation $E: A_{\alpha} \rightarrow C(T)$ by

$$
E(x)=\int_{\mathbf{T}} \hat{\alpha}_{t}(x) d t \quad\left(x \in A_{\alpha}\right),
$$

where $d t$ denotes normalized Haar measure on T. We have $E(f)=f(0)$ for $f$ in $C_{c}(\mathbf{Z}, C(\mathbf{T}))$. The algebra $A_{\alpha}$ is known to be simple $[\mathbf{1 2}, \mathbf{2 0}]$ and to have a unique normalized trace $\tau$. For $f$ in $C_{c}(\mathbf{Z}, C(\mathbf{T}))$ the trace is given by $f \rightarrow \int_{\mathbf{T}} f(0) d t$.

For $W$ a unitary on a Hilbert space $\mathcal{H}, B$ a $C^{*}$-algebra on $\mathcal{H}$, we let ad $W$ denote the *-automorphism of $B$ defined by $x \rightarrow W x W^{*}(x \in B)$.

If $V_{1}$ is the unitary in $C(\mathbf{T})$ defined by $t \rightarrow t(t \in \mathbf{T})$, then $U_{1} V_{1}=$ $\exp (2 \pi i \alpha) V_{1} U_{1}$. Suppose $U, V$ are two unitary operators satisfying this 
'twisted commutation relation'. As ad $U$ is a *automorphism of $C^{*}(V)$, the $C^{*}$-algebra generated by $V$, we have $\operatorname{sp}(V)$, the spectrum of $V$, is equal to $\operatorname{sp}(\operatorname{ad} U(V))=\exp (2 \pi i \alpha) \operatorname{sp}(V)$. Thus $\operatorname{sp}(V)=\mathbf{T}$ and $C(\mathbf{T}) \cong C^{*}(V)$, the ${ }^{*}$-isomorphism mapping $V_{1}$ to $V$. Using Proposition 1.2. and the fact that $A_{\alpha}$ is simple, we conclude that $A_{\alpha}$ is *-isomorphic to the $C^{*}$-algebra generated by $U$ and $V$, the isomorphism mapping $V_{1}$ to $V$ and $U_{1}$ to $U$. Dropping the subscripts from here on, we write $U$ and $V$ for $U_{1}, V_{1}$.

Let $X$ be a locally compact (2nd countable) Hausdorff space, $\mu$ a $\sigma$-finite positive Borel measure and $G$ a discrete countable group of homeomorphisms of $X$ (acting on the right by $x \rightarrow x s(x \in X, s \in G)$ ).

The measure $\mu$ is quasi-invariant under $G$ if $\mu(E)=0$ implies $\mu(E s)$ $=0$ ( $s \in G, E$ a measurable set). In this case the measure $\mu_{s}$ defined by $\mu_{s}(E)=\mu(E s)$ ( $E$ measurable) is absolutely continuous with respect to $\mu$ and the Radon-Nikodym derivative $d \mu_{s} / d \mu$ exists. The measure is invariant if $d \mu_{s} / d \mu \equiv 1$. The group action is free if $\mu(\{x \in X \mid x s=x\})=0$ for each $s$ in $G \backslash\{e\}, e$ the unit of $G$. The group action is ergodic if $f_{s}=f$ for all $s$ in $G$ implies $f$ is a constant a.e. $\left(f \in L^{\infty}(X, \mu)\right)$. Here $f_{s}(x)=f(x s)$ $(x \in X, s \in G$ ) for any function $f$ on $G$.

2. A class of representations of $A_{\alpha}$. A separable representation $\rho$ of $C(\mathbf{T})$ is of multiplicity $m, m \in N \cup\{\infty\}$, if $\rho(C(\mathbf{T}))^{\prime}$ is a type $\mathrm{I}_{m}$ von Neumann algebra. Given $\nu$ a regular Borel measure on $\mathbf{T}$, let $M_{f}$ denote the operator on the Hilbert space $L^{2}(\mathbf{T}, \nu)$ assigning $f g$ to $g ; g \in L^{2}(\mathbf{T}, \nu)$, $f \in L^{\infty}(\mathbf{T}, \nu)$. For $H_{m}$ a Hilbert space of dimension $m$, the representation $\rho_{m}$ of $C(\mathbf{T})$ defined by mapping $f$ to $M_{f} \otimes \operatorname{Id}_{H_{m}}$ is a uniform multiplicity $m$ representation on the Hilbert space $L^{2}(\mathbf{T}, \nu) \otimes H_{m}$. Conversely [10] if $\rho$ is a representation of $C(\mathbf{T})$ of uniform multiplicity $m$, there is a regular Borel measure $\nu$ on $\mathbf{T}$, uniquely determined up to equivalence of measures, such that $\rho$ is unitarily equivalent to the representation $\rho_{m}$ above.

We consider those separable representations $\pi$ of $A_{\alpha}$ whose restriction to $C(\mathbf{T})$ is of uniform multiplicity $m$, so we may suppose there is a regular Borel measure $\nu$ on $\mathbf{T}$ with $\left.\pi\right|_{C(\mathbf{T})}=\rho_{m}$. The measure $\nu$ is necessarily quasi-invariant under $\mathbf{Z}$, for if $f \in C(\mathbf{T})$ we have ad $\pi(U)\left(\rho_{m}(f)\right)=$ $\rho_{m}(\alpha(f))$, so $\rho_{m}(f)=0$ iff $\rho_{m}(\alpha(f))=0$. The regularity of $\nu$ ensures that $\nu_{\alpha} \sim \nu$.

Our interest in singling out those representations $\pi$ of $A_{\alpha}$ whose restriction to $C(\mathbf{T})$ is of uniform multiplicity lies in the fact that any factor representation is such. The argument ([11] §3.8) shows that the projections occurring in the central decomposition of the type I von Neumann algebra $\pi(C(\mathbf{T}))^{\prime}$ are invariant under ad $\pi(U)$ and so lie in the center of $\pi\left(A_{\alpha}\right)^{\prime \prime}$. A 
short argument shows that the measure $\nu$ in this case must be ergodic, for if $f \in L^{\infty}(\mathbf{T}, \nu)$ with $\alpha(f)=f$, then

$$
\text { ad } \pi(U)\left(M_{f} \otimes \mathrm{Id}_{H_{m}}\right)=M_{\alpha(f)} \otimes \operatorname{Id}_{H_{m}}=M_{f} \otimes \operatorname{Id}_{H_{m}} .
$$

Thus $M_{f} \otimes \mathrm{Id}_{H_{m}}$ lies in the center of $\pi\left(A_{\alpha}\right)^{\prime}$ and $f$ is constant (a.e. $\nu$ ).

Given $m$ in $\mathbf{N} \cup\{\infty\}$ and $\nu$ a quasi invariant regular Borel measure on $\mathbf{T}$, we may form a covariant representation $\left(\rho_{m}, \tilde{U} \otimes \mathrm{Id}_{H_{m}}\right)$ of $(C(\mathbf{T}), \alpha, \mathbf{Z})$ by

$$
\tilde{U} k=\sqrt{d \nu_{\alpha} / d \nu} \alpha(k) \quad\left(k \in L^{2}(\mathbf{T}, \nu)\right) \quad([\mathbf{1 7}, \mathbf{1 9}]) .
$$

When there is no possibility of confusion, we write $\tilde{U}$ instead of $\tilde{U} \otimes \operatorname{Id}_{H_{m}}$. Note that $n \rightarrow \operatorname{ad} \tilde{U}_{n}$ is then an action of $\mathbf{Z}$ by ${ }^{*}$-automorphisms on the $m \times m$ matrix algebra over $L^{\infty}(\mathbf{T}, \nu), \mathfrak{M}_{m}\left(L^{\infty}(\mathbf{T}, \nu)\right)$.

We recall ([19]) that a map $b$ of $\mathbf{Z}$ into the (non-abelian) group of unitaries in $\mathfrak{M}_{m}\left(L^{\infty}(\mathbf{T}, \nu)\right)$ is a unitary 1-cocycle if $b_{n+r}=b_{n}$ ad $\tilde{U}_{n}\left(b_{r}\right)$ for $n, r$ in $\mathbf{Z}$. Two such cocycles $b, c$ are equivalent if there is a unitary $Y$ in $\mathfrak{M}_{m}\left(L^{\infty}(\mathbf{T}, \nu)\right)$ with $b_{n}=Y^{-1} c_{n}$ ad $\tilde{U}_{n}(Y)$. With $m$ and $\nu$ fixed, there is a one-to-one correspondence between unitary equivalence classes of covariant representations $\left(\rho_{m}, T\right)$ of $(C(\mathbf{T}), \alpha, \mathbf{Z})$ and equivalence classes of unitary 1-cocycles $b$ of $\mathbf{Z}$ in $\mathfrak{M}_{m}\left(L^{\infty}(\mathbf{T}, \nu)\right)$ given by $T_{n}=b_{n}\left(\tilde{U}_{n} \otimes \operatorname{Id}_{H_{m}}\right)$ $(n \in \mathbf{Z})([\mathbf{1 9}])$.

Thus (unitary equivalence classes of) representations $\pi$ of $A_{\alpha}$ with $\left.\pi\right|_{C(\mathbf{T})}$ of uniform multiplicity are completely described by triples $(m, \nu, b)$ with $m$ in $N \cup\{\infty\}, \nu$ a quasi-invariant Borel measure (class) and $b$ a cocycle (equivalence class). In particular any factor representation of $A_{\alpha}$ may be described by such a triple.

Suited to the study of factor representations is the notion of quasiequivalence. Let $\pi$ and $\tilde{\pi}$ be two representations of a $C^{*}$-algebra $\mathfrak{A}$ and $\Re$, $\tilde{\Re}$ the von Neumann algebras generated by $\pi(\mathfrak{U}), \tilde{\pi}(\mathfrak{H})$, respectively. Recall $\pi$ is quasi-equivalent to $\tilde{\pi}$ (write $\pi \approx \tilde{\pi}$ ) if there is a ${ }^{*}$-isomorphism $\varphi: \Re \rightarrow \tilde{\mathscr{R}}$ with $\tilde{\pi}(x)=\varphi(\pi(x))$ for all $x$ in $\mathfrak{A}$, or, equivalently, if $\bigoplus^{c_{1}} \pi$ is unitarily equivalent to $\oplus^{c_{2}} \tilde{\pi}$ for some $c_{1}, c_{2} \in \mathbf{N} \cup\{\infty\}$ (for $\pi, \tilde{\pi}$ separable) [4].

If $\pi$ and $\tilde{\pi}$ are two separable factor representations of $A_{\alpha}$ that are quasi-equivalent, then we see (as a ${ }^{*}$-isomorphism of von Neumann algebras is weakly bicontinuous on the unit balls of the algebras) that $\left.\pi\right|_{C(\mathbf{T})}$ is quasi-equivalent to $\left.\tilde{\pi}\right|_{C(\mathbf{T})}$. Now these representations of $C(\mathbf{T})$ are of uniform multiplicity (as $\pi, \tilde{\pi}$ are factor representations). Thus the 
measures $\nu$ and $\tilde{\boldsymbol{\nu}}$ on $\mathbf{T}$ arising from these representations of $C(\mathbf{T})$ must be equivalent [10]. We can therefore assume, as far as quasi-equivalence of factor representations of $A_{\alpha}$ are concerned, that $\nu=\tilde{\nu}$.

Proposition 2.1. Let $\pi, \tilde{\pi}$ be two separable factor representations of $A_{\alpha}$. Let $\left(\rho_{m}, T\right)$ and $\left(\rho_{\tilde{m}}, \tilde{T}\right)$ be the associated covariant representations on $L^{2}(\mathbf{T}, \nu) \otimes H_{m}$ and $L^{2}(\mathbf{T}, \tilde{\nu}) \otimes H_{\tilde{m}}$, respectively, where $\nu, \tilde{\nu}$ are ergodic quasi-invariant regular Borel measures on $\mathbf{T}$ and $T_{n}=b_{n}\left(\tilde{U} \otimes \mathrm{Id}_{H_{m}}\right)_{n}, \tilde{T}_{n}=$ $\tilde{b}_{n}\left(\tilde{U} \otimes \mathrm{Id}_{H_{\tilde{m}}}\right)_{n}$ with $b_{n}, \tilde{b}_{n}$ unitary cocycles. Then $\pi \approx \tilde{\pi}$ iff $\nu \sim \tilde{\nu}$ ( so by the preceding comments we can assume $\nu=\tilde{\nu})$, and there exist $c_{1}, c_{2}$ in $\mathbf{N} \cup\{\infty\}$ with $m c_{1}=\tilde{m} c_{2}=r$ and a unitary $W$ in $\mathfrak{M}_{r}\left(L^{\infty}(\mathbf{T}, \nu)\right)$ on the Hilbert space $L^{2}(\mathbf{T}, \nu) \otimes H_{r}$ such that

$$
W\left(\stackrel{c_{1}}{\bigoplus} b_{1}\right)=\left(\stackrel{c_{2}}{\bigoplus} \tilde{b}_{1}\right) \operatorname{ad}\left(\tilde{U} \otimes \operatorname{Id}_{H_{r}}\right)(W) .
$$

Proof. We have $\pi \approx \tilde{\pi}$ iff there are $c_{1}, c_{2}$ with $m c_{1}=\tilde{m} c_{2}=r$ and a unitary $W$ on $L^{2}(\mathbf{T}, \nu) \otimes H_{r}$ with $W\left(\oplus^{c_{1}} \pi\right)=\left(\oplus^{c_{2}} \tilde{\pi}\right) W$. This is equivalent to

$$
W\left(\stackrel{c_{1}}{\bigoplus} \rho_{m}\right)=\left(\stackrel{c_{1}}{\bigoplus} \rho_{\tilde{m}}\right) W
$$

and

$$
W\left(\stackrel{c_{1}}{\bigoplus} b_{n}\left(\tilde{U} \otimes \mathrm{Id}_{H_{m}}\right)_{n}\right)=\left(\bigoplus^{c_{2}} \tilde{b}_{n}\left(\tilde{U} \otimes \mathrm{Id}_{H_{\tilde{m}}}\right)_{n}\right) W .
$$

The first equation means

$$
W \in \rho_{r}(C(\mathbf{T}))^{\prime}=\mathfrak{M}_{r}\left(L^{\infty}(\mathbf{T}, \nu)\right),
$$

and the second is equivalent to

$$
W\left(\stackrel{c_{1}}{\bigoplus} b_{1}\right)=\left(\bigoplus^{c_{2}} \tilde{b}_{1}\right) \operatorname{ad}\left(\tilde{U} \otimes \mathrm{Id}_{H_{r}}\right) W
$$

We return to examining covariant representations $\left(\rho_{m}, T\right)$ of $(C(\mathbf{T}), \alpha, \mathbf{Z})$, where $m \in \mathbf{N} \cup\{\infty\}$ and $\nu$ is a regular Borel measure of $\mathbf{T}$. We suppose from now on, without loss of generality, that $\nu(\mathbf{T})=1$. The next proposition shows that for $m$ finite we always have a type I representation. 
Proposition 2.2. Let $\pi$ denote the representation of $A_{\alpha}$ corresponding to a covariant representation $\left(\rho_{m}, T\right)$ of $(C(\mathbf{T}), \alpha, \mathbf{Z})$ where $m \in \mathbf{N}$ and $\nu$ is a regular Borel measure of $\mathbf{T}$. If $R$ is the von Neumann algebra $\left\{\pi(x) \mid x \in A_{\alpha}\right\}^{\prime \prime}$, then $\Re$ is type I. Furthermore there are central projections $P_{s}, s \in\{1, \ldots, m\}$, of $\Re$ with $\Sigma P_{s}=1$ and $\Re^{\prime} P_{s}$ of type $\mathrm{I}_{s}$ or $P_{s}=0$.

Proof. To show $\mathcal{R}$ is of type $\mathrm{I}$ it is enough to show that $\mathcal{R}^{\prime}$ is type I. If a bounded operator $C$ on $L^{2}(\mathbf{T}, \nu) \otimes H_{m}$ lies in $\Re^{\prime}$, then $C \in \rho_{m}(C(\mathbf{T}))^{\prime}=$ $\mathfrak{M}_{m}\left(L^{\infty}(\mathbf{T}, \boldsymbol{\nu})\right)$. Thus $\mathfrak{R}^{\prime} \subseteq \mathfrak{M}_{m}\left(L^{\infty}(\mathbf{T}, \boldsymbol{\nu})\right)$, which is a type $\mathrm{I}_{m}$ von Neumann algebra. It is well known that $\Omega^{\prime}$ must then be a direct sum of type $\mathrm{I}_{s}(s \leq m)$ von Neumann algebras ( $\Omega^{\prime}$ inherits a faithful scalar trace from $\left.\mathfrak{M}_{m}\left(L^{\infty}(\mathbf{T}, \nu)\right)\right)$.

Let $\pi$ be a representation of $A_{\alpha}$ with $\left.\pi\right|_{C(\mathbf{T})}$ a uniform multiplicity representation. Conditions on the triple $(m, \nu, b)$ associated with $\pi$ are reflected in the structure of the von Neumann algebra $\Re=\pi\left(A_{\alpha}\right)^{\prime \prime}$. We have seen, for example, that $\Re$ is type I if $m<\infty$. Another case considered below is when $\nu$ is ergodic and $b$ is a "diagonal cocycle". In this case $\Re$ will be type I and we can say exactly when $R$ is a factor by examining an equivalence relation on $\{1, \ldots, m\}$. Note $m=\infty$ is not excluded in these considerations.

We say an operator in $\mathfrak{M}_{m}\left(L^{\infty}(\mathbf{T}, \nu)\right.$ ) is diagonal if it lies in the (von Neumann) subalgebra $\bigoplus^{m} L^{\infty}(\mathbf{T}, \boldsymbol{v})$. A unitary cocycle $b$ of $\mathbf{Z}$ in $\mathfrak{M}_{m}\left(L^{\infty}(\mathbf{T}, \nu)\right)$ will be called diagonal if $b_{1}$ is diagonal.

LEMMA 2.3. Let $\pi$ be a representation of $A_{\alpha}$ corresponding to a triple $(m, \nu, b)$ where, in addition, $\nu$ is ergodic and $b$ is a diagonal cocycle. Let $b_{1}=\oplus^{m} \lambda$, with $\lambda_{j} \in L^{\infty}(\mathbf{T}, \mu)$ and let $\Re=\pi\left(A_{\alpha}\right)^{\prime \prime}$. We have a relation $\sim$ on $\{r \in \mathbf{N} \mid 1 \leq r \leq m\}$ if $m<\infty$ and on $\mathbf{N} \backslash\{0\}$ if $m=\infty$ by defining $r \sim s$ iff there is a non-zero element $d$ of $L^{\infty}(\mathbf{T}, \nu)$ with $\alpha(d)=\bar{\lambda}_{r} \lambda_{s} d$. The following hold.

(1) The relation $\sim$ is an equivalence relation.

(2) If $C \in \Re^{\prime}$ and $j \nsim k$ then the jk entry of $C$ (viewed as a matrix in $\left.\mathfrak{B}\left(L^{2}(\mathbf{T}, \nu) \otimes H_{m}\right)\right), c_{j k}$, is zero.

(3) If $A \in \Re \cap \Re^{\prime}$, the center of $\Re$, and $j \sim k$ with $j \neq k$, then $a_{J J}=a_{k k}$ and $a_{J k}=0$.

Proof. (1) Call $d$ a " $(j, k)$ solution" if $d$ is a non-zero element of $L^{\infty}(\mathbf{T}, \nu)$ with $\alpha(d)=\bar{\lambda}_{J} \lambda_{k} d$. As $b$ is unitary, we have $\left|\lambda_{J}\right|=1$, so $j \sim j$, as 
constants are $(j, j)$ solutions. If $d$ is a $(j, k)$ solution, $\bar{d}$ is a $(k, j)$ solution. Note that $|d|^{2}=d \bar{d}$ is a $(k, k)$ solution and thus a (non-zero) constant as $\nu$ is ergodic. Thus if $d$ and $h$ are $(j, k),(k, r)$ solutions, respectively, $d h$ is non-zero and so a $(j, r)$ solution.

(2) A bounded operator $C$ on $L^{2}(\mathbf{T}, \nu) \otimes H_{m}$ lies in $\Omega^{\prime}$ if $C \in$ $\rho_{m}(C(\mathbf{T}))^{\prime}=\mathfrak{M}_{m}\left(L^{\infty}(\mathbf{T}, \nu)\right)$ and $\operatorname{ad}\left(b_{1} \tilde{U}\right)(C)=C$. The latter equation holds iff ad $\tilde{U}(C)=b_{1}^{*} C b_{1}$ iff $\alpha\left(c_{\jmath k}\right)=\bar{\lambda}_{j} \lambda_{k} c_{j k}$ for each $j, k$. By definition $c_{j k}=0$ if $j \nsim k$.

(3) Let $d$ be a $(j, k)$ solution. We define an element $C$ of $\mathfrak{M}_{m}\left(L^{\infty}(\mathbf{T}, \nu)\right)$ as follows: the $j, k$ entry of $C$ is $d, c_{J J}, c_{k k}$ are two arbitrary constants and $c_{r s}=0$ for all other $r$, s. As $\alpha\left(c_{r s}\right)=\bar{\lambda}_{r} \lambda_{s} c_{r s}$ for all $r, s, C \in \Re^{\prime}$. As $A$ commutes with the elements $C$, we have, upon examining $j, k$ entries from the matrix equation $A C=C A,\left(a_{j j}-a_{k k}\right) c_{j k}=a_{j k}\left(c_{j J}-c_{k k}\right)$. We may vary the constant $c_{J j}-c_{k k}$ at will, while the left side of the equation remains unchanged. Thus $a_{j k}=0$ (a.e. $\nu$ ). Now $c_{j k}=d$ is non-zero and $a_{J J}, a_{k k}$ are constants (as $A \in R^{\prime}$ ). Thus $a_{J J}-a_{k k}=0$.

Proposition 2.4. With the hypothesis of the previous lemma, the following are true.

(1) If $k \nsim j$ for all $k \neq j$ then $\Re^{\prime}=\oplus^{m} \mathbf{C}$ so $\Re=\oplus^{m} \mathfrak{B}\left(L^{2}(\mathbf{T}, \nu)\right)$.

(2) If $k \sim j$ for all $k, j$ then $\Re^{\prime}$ is a type $\mathrm{I}_{m}$ factor.

(3) If $r \nsim s$, some $r \neq s$, then $R$ is not a factor.

(4) $\Re$ is type I.

Proof. (1) For $C$ in $\mathcal{R}^{\prime}$ we have $c_{k k}$ is a constant for each $k$ as $\nu$ is ergodic. By the lemma, $c_{r s}=0$ for all $r, s$ with $r \neq s$. Thus $\Re^{\prime}=\bigoplus^{m} \mathbf{C}$ and $R=\oplus^{m} \mathfrak{B}\left(L^{2}(\mathbf{T}, \boldsymbol{\nu})\right)$.

(2) The lemma shows for $A$ in $\Re \cap \Re^{\prime}$ that $a_{r s}=0$ and $a_{r r}=a_{s s}$ (is a constant) for all $r, s$ with $r \neq s$. Thus $\Re$ is a factor. Let $E$ be the element of $\mathfrak{M}_{m}\left(L^{\infty}(\mathbf{T}, \nu)\right)$ described by defining all entries $e_{r s}$ to be zero except the $(j, k)$ entry which is a $(j, k)$ solution $d$ with $|d|=1$ (a.e. $\nu)$. Again, as $\alpha\left(e_{r s}\right)=\bar{\lambda}_{r} \lambda_{s} e_{r s}, E$ is in $\Omega^{\prime}$. The $(k, k)$ entry of $E^{*} E, d \bar{d}$, is equal to 1 (a.e. $\nu$ ), while all other entries are zero. Thus $E$ is a partial isometry with initial projection $P_{k}$ and final projection $P_{j}$. Here $P_{r}$ denotes the projection in $\Re^{\prime}$ that has all zero entries except the $(r, r)$ diagonal entry, which is 1 . The projection $P_{r}$ is abelian in $\mathfrak{M}_{m}\left(L^{\infty}(\mathbf{T}, \nu)\right)$, thus abelian in $\Re^{\prime}$. As $\sum_{1}^{m} P_{r}=\mathrm{Id}, \Re^{\prime}$ is type $\mathrm{I}_{m}$.

(3) We display non-scalar elements $A$ in the center of $R$. If $h_{1}, h_{2}$ are two distinct constants, define $a_{J j}=h_{1}$ if $r \sim j, a_{j J}=h_{2}$ if $j \sim s$ and $a_{p q}=0$, all other $p, q$. As $r \nsim s, a_{j J}$ is well defined. Note that $A$ is not a 
scalar and, since $\oplus^{m} \mathbf{C} \subseteq \Re^{\prime}, A \in \Re^{\prime}$. To conclude that $A \in \Re$, we prove $A C=C A$ for all $C$ in $\Re^{\prime}$. We show that the $(p, q)$ entry of $A C$,

$$
a_{p p} c_{p q}= \begin{cases}h_{1} C_{p q} & \text { iff } r \sim p \\ h_{2} C_{p q} & \text { iff } p \sim s \\ 0 & \text { otherwise }\end{cases}
$$

is equal to the $(p, q)$ entry of $C A$,

$$
c_{p q} a_{q q}= \begin{cases}h_{1} C_{p q} & \text { iff } r \sim q, \\ h_{2} C_{p q} & \text { iff } q \sim s, \\ 0 & \text { otherwise. }\end{cases}
$$

If $r \sim p$ and $r \nsim q$, then $p \nsim q$ so $c_{p q}=0$. Similarily, if $p \sim s$ and $q \nsim s$, then $p \nsim q$ and $c_{p q}=0$, and we have $a_{p p} c_{p q}=c_{p q} a_{q q}$. If both $r \nsim p$ and $p \nsim s$, then we must show $c_{p q} a_{q q}=0$. This can be non-zero only if $r \sim q$ or $q \sim s$. In both cases, $p \nsim q$ (otherwise $r \sim p$ or $p \sim s$, contradicting our present assumption). Thus $c_{p q}=0$ and $c_{p q} a_{q q}=0$.

(4) Changing to an equivalent cocycle by a permutation unitary, we may assume that if $j \sim k$, then $j \sim s$ for all $s$ with $j \leq s \leq k$. Combining the arguments in (2) and (3) shows $\mathcal{R}^{\prime}$ is a direct sum of type I factors.

An example where $\Re$ is a factor is $b_{1}=\mathrm{Id}$. For an example with $\Re$ not a factor let $\nu=$ Haar measure on $\mathbf{T}$ and $b_{1}=\left[\begin{array}{cc}-1 & 0 \\ 0 & 1\end{array}\right]$. Then $1 \nsim 2$, for if $d \in L^{\infty}(\mathbf{T}, \nu)$ with $\alpha(d)=-d$, then $\alpha_{2}(d)=d$. As $2 \alpha \bmod 1$ is still irrational, $d$ is a constant. As $d=-d, d=0$.

Suppose the 1-cocycle $b$ is cohomologous to a diagonal cocycle, i.e., there is a unitary $Y$ in $\mathfrak{M}_{m}\left(L^{\infty}(\mathbf{T}, \nu)\right)$ such that $Y^{-1} b_{n}$ ad $\tilde{U}_{n}(Y)$ is a diagonal cocycle. Then the covariant representation $\left(\rho_{m}, T\right)$ is unitarily equivalent to a covariant representation with a diagonal cocycle. A result of Kadison [9] shows that if $m$ is finite, there is a unitary $W$ in $\mathfrak{M}_{m}\left(L^{\infty}(\mathbf{T}, \nu)\right)$ such that $W^{*} b_{1} W$ is diagonal. Note that, in general, this is not enough to ensure that our cocycle is cohomologous to a diagonal cocycle. However, if the unitary $W$ had constant entries, i.e., $W \in \mathfrak{M}_{m}(\mathbf{C})$ $\otimes \operatorname{Id}_{L^{2}(\mathbf{T}, \nu)}$, then ad $\tilde{U}_{n}(W)=W$ and the cocycle $b$ is cohomologous to a diagonal cocycle. If $m$ is finite and $b_{1}$ is a unitary in $\mathfrak{M}_{m}(\mathbf{C}) \otimes \operatorname{Id}_{L^{2}(\mathbf{T}, \nu)}$, such a unitary $W$ with constant entries always exists.

If the measure $\nu$ is ergodic, the unitary $b_{1}$ has constant entries iff $T_{1}$ commutes with $\tilde{U}_{1}$. This follows from the fact that $T_{1}$ commutes with $\tilde{U}_{1}$ iff ad $\tilde{U}_{1}\left(b_{1}\right)=b_{1}$ iff $\alpha\left(\left(b_{1}\right)_{j k}\right)=\left(b_{1}\right)_{j k}$ for each $j, k$. 
3. Automorphisms of $A_{\alpha}$. A representation of the discrete group $\mathrm{SL}(2, \mathbf{Z})$ as automorphisms of $A_{\alpha}$ arises naturally when viewing $A_{\alpha}$ as a twisted covariance algebra [6]. We also investigate a few simple properties of the group homomorphism of $\operatorname{Aut}\left(A_{\alpha}\right)$ to $\operatorname{Aut}\left(K_{1}\left(A_{\alpha}\right)\right)$ defined by mapping an automorphism $\gamma$ of $A_{\alpha}$ to $\gamma_{*}$, the induced isomorphism of the abelian group $K_{1}\left(A_{\alpha}\right)$. We show that the kernel of this map, $\gamma \rightarrow \gamma_{*}$, is closed and contains the centrally trivial automorphisms.

If $G$ is a separable locally compact group and $\mathbf{T}$ the circle group, a Borel function $\omega$ from $G \times G$ to $\mathbf{T}$ is a multiplier (a 2-cocycle on $G$ with coefficients in $\mathbf{T})[\mathbf{1 1}]$ if

(a) $\omega(x, y) \omega(x y, z)=\omega(x, y z) \omega(y, z),(x, y, z) \in G$;

(b) $\omega(x, e)=\omega(e, x)=1$ with $e$ the unit of $G, x \in G$.

Given a multiplier $\omega$ on $G$, an $\omega$-representation of $G$ is a strongly continuous map $L$ of $G$ to the unitary group on a separable Hilbert space with $L_{e}=\operatorname{Id}$ and $L_{x} L_{y}=\omega(x, y) L_{x y}$ for $x, y$ in $G$.

Define a multiplier on the discrete group $G=\mathbf{Z} \times \mathbf{Z}$ by

$$
\omega\left((m, n),\left(m^{\prime}, n^{\prime}\right)\right)=\exp \left[\pi i \alpha\left(m^{\prime} n-m n^{\prime}\right)\right]=\exp \left[\pi i \alpha\left|\begin{array}{cc}
m^{\prime} & m \\
n^{\prime} & n
\end{array}\right|\right]
$$

where $\left[\begin{array}{ll}m^{\prime} & m \\ n^{\prime} & n\end{array} \mid\right.$ denotes the determinant of the matrix $\left[\begin{array}{cc}m^{\prime} & m \\ n^{\prime} & n\end{array}\right], m, n, m^{\prime}, n^{\prime}$ in $\mathbf{Z}$. For $g$ in $\operatorname{SL}(2, \mathbf{Z})$, we note $\omega\left(g(m, n), g\left(m^{\prime}, n^{\prime}\right)\right)=\omega\left((m, n)\left(m^{\prime}, n^{\prime}\right)\right)$, where $\operatorname{SL}(2, \mathbf{Z})$ acts on $\mathbf{Z} \times \mathbf{Z}$ in the usual manner. We form the Mackey obstruction group, a locally compact separable group $G(\omega)$ with underlying set $\{(x, t) \mid x \in G, t \in \mathbf{T}\}$ and multiplication given by $(x, t)\left(y, t^{\prime}\right)=$ $\left(x y, \omega(x, y) t t^{\prime}\right)$ for $x, y$ in $G$ and $t, t^{\prime}$ in $\mathbf{T}$. The usefulness of $G(\omega)$ lies in the fact that the $\omega$-representations of $G, L$, are in one-to-one correspondence with the unitary representations $W$ of $G(\omega)$ restricting on $T$ to a multiple of the one-dimensional representation $t \rightarrow t$. The correspondence is given by $W(x, t)=t L_{x}[\mathbf{1 1}]$.

Let $C_{c}(G(\omega), \mathbf{T})$ be the set of functions from $G(\omega)$ to $\mathbf{C}$ with $f(t x)=$ $t^{-1} f(x)(t \in \mathbf{T}, x \in G(\omega))$ such that $f(m, n, t)$ is non-zero for only finitely many $m, n$ in $\mathbf{Z}$. Define a normed *-algebra structure on $C_{c}(G(\omega), \mathbf{T})$ as follows: for $f, h$ in $C_{c}(G(\omega), \mathbf{T})$,

$$
\begin{gathered}
\|f\|=\sum_{m, n \in \mathbf{Z}}|f(m, n, 1)|, \\
f^{*}(m, n, t)=\overline{f\left(-m,-n, t^{-1}\right)} \\
f * h(m, n, t)=\sum_{a, b \in \mathbf{Z}} f(a, b, 1) h[(-a,-b, 1)(m, n, t)] .
\end{gathered}
$$


The enveloping $C^{*}$-algebra of the completion of this normed *-algebra, denoted $C^{*}(G(\omega), \mathbf{T})$, is a twisted covariance algebra. There is a one-to-one correspondence between *-representations of $C^{*}(G(\omega), \mathbf{T})$ and representations of $G(\omega)$ restricting to a multiple of $t \rightarrow t$ on T [6].

The $C^{*}$-algebra $C^{*}(G(\omega), \mathbf{T})$ is *-isomorphic to $A_{\alpha}$. If $f_{(m, n)}$ is the element of $C_{c}(G(\omega), \mathbf{T})$ defined by mapping all elements to zero except $(m, n, t)$, which is mapped to $t^{-1} \xi(m, n)$, where $\xi(m, n)=\exp (\pi i \alpha m n)$, we have

$$
f_{(m, n)} * f_{(\tilde{m}, \tilde{n})}=\exp (2 \pi i \alpha \tilde{m} n) f_{(m+\tilde{m}, n+\tilde{n})} .
$$

Thus $f_{(1,0)}$ and $f_{(0,1)}$ are two unitaries $V, U$, respectively, with $U V=e^{2 \pi i \alpha} V U$. The $C^{*}$-algebra generated by $f_{(1,0)}$ and $f_{(0,1)}$ is $C^{*}(G(\omega), \mathbf{T})$. Thus $C^{*}(G(\omega), \mathbf{T}) \cong A_{\alpha}$.

For $g$ in $\operatorname{SL}(2, \mathbf{Z})$ let $g(m, n)=(\tilde{m}, \tilde{n})$ denote the usual action of $\operatorname{SL}(2, \mathbf{Z})$ on $\mathbf{Z} \times \mathbf{Z}$. Let $\gamma_{g}$ be a map of $G(\omega)$ to $G(\omega)$ defined by $\gamma_{g}(m, n, t)=(g(m, n), t) \quad(g \in \mathrm{SL}(2, \mathbf{Z}))$. As $\omega(g(m, n), g(p, q))=$ $\omega((m, n)(p, q))$ for $m, n, p, q$ in $\mathbf{Z}, \gamma_{g}$ is a group automorphism of $G(\omega)$. The relation $\gamma_{g_{1} g_{2}}=\gamma_{g_{1}} \gamma_{g_{2}}$ for $g_{1}, g_{2}$ in $\operatorname{SL}(2, \mathbf{Z})$ shows that $\gamma: \operatorname{SL}(2, \mathbf{Z}) \rightarrow$ Aut $_{\text {group }}(G(\omega))$ is a group homomorphism. Define an action of $\operatorname{SL}(2, \mathbf{Z})$ by isometric *-automorphisms of the normed *-algebra $C_{c}(G(\omega), \mathbf{T})$ by ${ }^{g} f(x)=f\left(\gamma_{g^{-1}}(x)\right)$ for $f$ in $C_{c}(G(\omega), \mathbf{T}), g$ in $\operatorname{SL}(2, \mathbf{Z}), x$ in $G(\omega)$. This action extends to an action $g \rightarrow \beta_{g^{\prime}}$ of $\operatorname{SL}(2, \mathbf{Z})$ by *automorphisms of the enveloping $C^{*}$-algebra $A_{\alpha}$. For later use we note that

$$
\begin{aligned}
\beta_{g}\left(V^{m} U^{n}\right) & =\beta_{g}\left(f_{(m, n)}\right)=\xi(m, n)^{-1} \xi(\tilde{m}, \tilde{n}) f_{(\tilde{m}, \tilde{n})} \\
& =\xi(m, n)^{-1} \xi(\tilde{m}, \tilde{n}) V^{\tilde{m}} U^{\tilde{n}} .
\end{aligned}
$$

If $\mathfrak{A}$ is a $C^{*}$-algebra with unit, let $U_{n}(\mathfrak{U})$ be the unitary group of $\mathfrak{M}_{n}(\mathfrak{U})$ and $U_{n}^{0}(\mathfrak{U})$ the connected component of the Id in $U_{n}(\mathfrak{U})$. We define $K_{1}(\mathfrak{A})=\lim _{\rightarrow} U_{n}(\mathfrak{A}) / U_{n}^{0}(\mathfrak{A})$, the direct limit of the direct system of groups $\left(\vec{U}_{n}(\mathfrak{A}) / U_{n}^{0}(\mathfrak{A}), \varphi_{n}\right)$, where $\varphi_{n}: \quad U_{n}(\mathfrak{A}) / U_{n}^{0}(\mathfrak{U}) \rightarrow$ $U_{n+1}(\mathfrak{A}) / U_{n+1}^{0}(\mathfrak{A})$ is given by $x \rightarrow\left(\begin{array}{ll}x & 0 \\ 0 & 1\end{array}\right)$. For $d$ in $U_{n}(\mathfrak{A})$ we denote by $[d]$ its image in $K_{1}(\mathfrak{U}) . K_{1}$ is a functor from the category of $C^{*}$-algebras (with unit, although this is not necessary) to the category of abelian groups [18].

Let $U, V$ be two unitary operators with $U V=e^{2 \pi i \alpha} V U$ that generate the $C^{*}$-algebra $A_{\alpha}$. It is known ([13]) that $K_{1}\left(A_{\alpha}\right)=\mathbf{Z} \oplus \mathbf{Z}$, where $[U]$, $[V]$ correspond to $(0,1)$ and $(1,0)$, respectively. Examining the automorphisms $\beta_{g}(g \in \operatorname{SL}(2, \mathbf{Z}))$ of $A_{\alpha}$, it is clear that $\left(\beta_{g}\right)_{*}$ is just the map $g$ on $\mathbf{Z} \oplus \mathbf{Z}$.

Putting the topology of pointwise norm convergence on $\operatorname{Aut}\left(A_{\alpha}\right)$ (a net $\gamma_{j} \rightarrow \gamma$ iff $\left\|\gamma_{j}(x)-\gamma(x)\right\| \rightarrow 0$ for each $x$ in $A_{\alpha}$ ), we have that the 
subgroup $\left\{\gamma \in \operatorname{Aut}\left(A_{\alpha}\right) \mid \gamma_{*}=\operatorname{Id}\right\}$ is closed. To see this, suppose $\gamma_{j} \rightarrow \gamma$ in $\operatorname{Aut}\left(A_{\alpha}\right)$ with $\left(\gamma_{j}\right)_{*}=$ Id for all $j$. Then there is a $j_{0}$ with $\| \gamma_{j}(U)-$ $\gamma(U) \|<\varepsilon$ and $\left\|\gamma_{j_{0}}(V)-\gamma(V)\right\|<\varepsilon$, where $\varepsilon$ is chosen small enough to ensure that $\gamma_{j_{0}}(U)$ (resp. $\gamma_{j_{0}}(V)$ ) lie in the path component of $\gamma(U)$ (resp. $\gamma(V))$ in $U_{1}\left(A_{\alpha}\right)$. Thus $\left[\gamma_{j_{0}}(U)\right]=[\gamma(U)]$ and $[U]=\left(\gamma_{j_{0}}\right)_{*}[U]=[\gamma(U)]$. It is also seen that $[V]=[\gamma(V)]$ and thus $\gamma_{*}=$ Id.

Definition. A norm bounded sequence $\left\{x_{n}\right\}$ in a $C^{*}$-algebra $\mathfrak{A}$ is (norm) central sequence if $\left\|x_{n} a-a x_{n}\right\| \rightarrow 0$ as $n \rightarrow \infty$ for each $a$ in $\mathfrak{A}$. An automorphism $\gamma$ of $\mathfrak{U}$ is centrally trivial (i.e., $\gamma \in \mathrm{Ct}(\mathfrak{A}))$ iff $\left\|\gamma\left(x_{n}\right)-x_{n}\right\|$ $\rightarrow 0$ as $n \rightarrow \infty$ for each central sequence $x_{n}$ in $\mathfrak{A}$ [3], [7].

Note that if $\gamma \in \operatorname{Aut}(\mathfrak{U})$ and $\left\{x_{n}\right\}$ is a central sequence, then $\left\{\gamma\left(x_{n}\right)\right\}$ is again a central sequence. It follows that $\mathrm{Ct}(\mathfrak{U})$ is a subgroup of $\operatorname{Aut}(\mathfrak{U})$. Also $\mathrm{Ct}(\mathfrak{U})$ contains $\operatorname{Int}(\mathfrak{U})$, the group of inner automorphisms of $\mathfrak{A}$, for if $W$ is a unitary in $\mathfrak{A}$ with $\gamma(a)=W a W^{*}(a \in \mathfrak{A})$, then

$$
\left\|\gamma\left(x_{n}\right)-x_{n}\right\|=\left\|W x_{n} W^{*}-x_{n}\right\|=\left\|W x_{n}-x_{n} W\right\| \rightarrow 0 \quad(n \rightarrow \infty)
$$

for any central sequence $x_{n}$.

In the $C^{*}$-algebra $A_{\alpha}$ there are ready examples of central sequences. If $p_{j} \in \mathbf{N} \backslash\{0\}$ is a sequence with $e^{2 \pi i p_{j} \alpha} \rightarrow 1$ as $j \rightarrow \infty$, then both $U^{p_{j}}, V^{p_{j}}$ are central sequences. For example

$$
\left\|U^{p_{J}} V-V U^{p_{J}}\right\|=\left\|U^{p_{J}} V U^{-p_{J}}-V\right\|=\left\|\left(e^{2 \pi p_{p_{J}} \alpha}-1\right) V\right\| \rightarrow 0 \quad \text { as } j \rightarrow \infty .
$$

Thus $\left\|U^{p_{J}} x-x U^{p_{J}}\right\| \rightarrow 0$ for $x$ in the *algebra generated by $U$ and $V$, and as this algebra is dense in $A_{\alpha}$, this holds for all $x$ in $A_{\alpha}$.

\section{Proposition 3.1. If $\gamma \in \operatorname{Ct}\left(A_{\alpha}\right)$ then $\gamma_{*}=$ Id.}

Proof. Fix a sequence $p_{j} \in \mathbf{N} \backslash\{0\}$ with $e^{2 \pi i p_{j} \alpha} \rightarrow 1$ as $j \rightarrow \infty$. As $\gamma$ is in $\operatorname{Ct}\left(A_{\alpha}\right)$, we must have $\left\|\gamma\left(U^{p_{J}}\right)-U^{p_{J}}\right\| \rightarrow 0$ and $\left\|\gamma\left(V^{p_{J}}\right)-V^{p_{J}}\right\| \rightarrow 0$ as $j \rightarrow \infty$. Thus for $j$ large enough, $\gamma\left(U^{p_{J}}\right) U^{-p_{J}} \in U_{1}^{0}\left(A_{\alpha}\right)$, so

$$
[1]=\left[\gamma\left(U^{p_{J}}\right) U^{-p_{J}}\right]=p_{j}\left(\left[\gamma_{*}(U)\right]-[U]\right) .
$$

As $K_{1}\left(A_{\alpha}\right)$ is torsion free, $\gamma_{*}[U]=[U]$. Similarly, $\gamma_{*}[V]=[V]$.

We have seen that $\left(\beta_{g}\right)_{*}$ is the map $g$ on $\mathbf{Z} \oplus \mathbf{Z}$. Thus $\beta_{g}$ is not in the closure of $\mathrm{Ct}\left(A_{\alpha}\right)$ for $g \neq \mathrm{Id}$.

An interesting question is whether the image of the map $\varphi \rightarrow \varphi_{*}$ of $\operatorname{Aut}\left(A_{\alpha}\right)$ to $\operatorname{Aut}(\mathbf{Z} \times \mathbf{Z})$ is all of $\operatorname{GL}(2, \mathbf{Z})$ or exactly $\operatorname{SL}(2, \mathbf{Z})$. In other words, is there an automorphism $\varphi$ of $A_{\alpha}$ with $\varphi_{*}$ of determinant -1 , in 
particular with $\varphi_{*}=\left[\begin{array}{ll}0 & 1 \\ 1 & 0\end{array}\right]$ ? Rewording this amounts to asking if there are two unitaries, $\varphi(U)$ and $\varphi(V)$ say, of $A_{\alpha}$ with commutator [ $\varphi(U), \varphi(V)$ ] $\left(=\varphi(U) \varphi(V) \varphi(U)^{-1} \varphi(V)^{-1}\right)=\exp (2 \pi i \alpha)$, and such that the equivalence class $[\varphi(U)]=[V]$ and $[\varphi(V)]=[U]$ (where $U, V$ are generating unitaries of $A_{\alpha}$ with $\left.[U, V]=\exp (2 \pi i \alpha)\right)$.

4. Pure states of $A_{\alpha}$. We define for each $\theta$ in $[0,1]$ a pure state $\varphi_{\theta}$ of $A_{\alpha}$. We show $\varphi_{\theta}$ is unitarily equivalent to $\varphi_{\theta^{\prime}}$ (i.e., there is a unitary $W$ in $A_{\alpha}$ with $\varphi_{\theta^{\prime}} \circ$ ad $\left.W=\varphi_{\theta}\right)$ iff $e^{2 \pi i \theta^{\prime}} \in\left\{e^{2 \pi i(\theta+n \alpha)} \mid n \in \mathbf{Z}\right\}$ (that is, iff $\theta^{\prime}$ and $\theta$ lie in the same orbit of the $\alpha$ action of $\mathbf{Z}$ on $\mathbf{T}$ ), and also state similar conditions for $\varphi_{\theta}, \circ \beta_{g}$ to be unitarily equivalent to $\varphi_{\theta}$ (for $g$ in $\mathrm{SL}(2, \mathbf{Z}))$. We show, too, that the set of pure states $\left\{\varphi_{\theta} \circ \beta_{g} \mid \theta \in[0,1]\right.$, $g \in \operatorname{SL}(2, \mathbf{Z})\}$ separate the points of $A_{\alpha}$.

Recalling the conditional expectation $E: A_{\alpha} \rightarrow C(\mathbf{T})$, we define a linear functional $\varphi_{\theta}=\rho_{\theta} \circ E$ for $\theta$ in $[0,1]$ where $\rho_{\theta}$ is the (pure) state of $C(\mathbf{T})$ given by evaluation at the point $e^{2 \pi i \theta}$ of $\mathbf{T}$. $A s \varphi_{\theta}(1)=1$ and

$$
\varphi_{\theta}\left(x^{*} x\right)=\rho_{\theta}\left(E\left(x^{*} x\right)\right)=\int_{\mathbf{T}} \rho_{\theta}\left(\hat{\alpha}_{t}\left(x^{*} x\right)\right) d t \geq 0
$$

(since $\rho_{\theta}$ is positive), $\varphi_{\theta}$ is a state. The following proposition also follows from more general considerations [2].

Proposition 4.1. The state $\varphi_{\theta}$ is a pure state of $A_{\alpha}$.

Proof. We show that if $\eta$ is a positive linear functional with $0 \leq \eta \leq \varphi_{\theta}$, then $\eta=\lambda \varphi_{\theta}$ for some $\lambda$ in C. For $\varphi$ a positive linear functional on $A_{\alpha}$, let $N(\varphi)=\left\{x \in A_{\alpha} \mid \varphi\left(x^{*} x\right)=0\right\}$, the left kernel of $\varphi$. If $K$ denotes the dense subalgebra $C_{c}(\mathbf{Z}, C(\mathbf{T}))$ of $A_{\alpha}$, we show

$$
K \cap \varphi_{\theta}^{-1}(0) \subseteq K \cap N\left(\varphi_{\theta}\right)+K \cap N\left(\varphi_{\theta}\right)^{*} .
$$

If $(*)$ is true then

$$
\begin{aligned}
K \cap \varphi_{\theta}^{-1}(0) & \subseteq K \cap N\left(\varphi_{\theta}\right)+K \cap N\left(\varphi_{\theta}\right)^{*} \\
& \subseteq K \cap N(\eta)+K \cap N(\eta)^{*} \subseteq K \cap \eta^{-1}(0),
\end{aligned}
$$

the last inclusion following from the Schwarz inequality. Thus $K \cap \varphi_{\theta}^{-1}(0)$ $\subseteq K \cap \eta^{-1}(0)$ and $\left.\eta\right|_{K}=\left.\lambda \varphi_{\theta}\right|_{K}$ for some $\lambda \in \mathbf{C}$. Continuity yields $\eta=\lambda \varphi_{\theta}$.

We prove the inclusion $(*)$.

$$
\begin{aligned}
K \cap N\left(\varphi_{\theta}\right) & =\left\{k \in K \mid \rho_{\theta}\left(k^{*} k(0)\right)=0\right\} \\
& =\left\{k \in K \mid \varphi_{\theta}\left(\sum \alpha_{n}\left(|k(-n)|^{2}\right)\right)=0\right\} \\
& =\left\{k \in K \mid k(-n)\left(e^{2 \pi i(\theta+n \alpha)}\right)=0, n \in \mathbf{Z}\right\} .
\end{aligned}
$$


Also

$$
\begin{aligned}
K \cap N\left(\varphi_{\theta}\right)^{*} & =\left\{k \in K \mid \varphi_{\theta}\left(k k^{*}(0)\right)=0\right\} \\
& =\left\{k \in K \mid k(n)\left(e^{2 \pi i \theta}\right)=0, n \in \mathbf{Z}\right\} .
\end{aligned}
$$

Now fix a $k$ in $K \cap \varphi_{\theta}^{-1}(0)$. As $e^{2 \pi i(\theta+n \alpha)} \neq e^{2 \pi i \theta}$ for all $n$ in $\mathbf{Z} \backslash\{0\}$, there are $f(n), g(n)$ in $C(\mathbf{T})$ with $k(n)=f(n)+g(n)$ and $f(n)\left(e^{2 \pi i \theta}\right)=$ $g(n)\left(e^{2 \pi t(\theta-n \alpha)}\right)=0$ for all $n \in \mathbf{Z} \backslash\{0\}$. For example, if $n \in \mathbf{Z} \backslash\{0\}$ let $g(n)=k(n) h(n)$ and $f(n)=k(n)(1-h(n))$, where $h(n)$ is an element of $C(\mathbf{T})$ with $h(n)\left(e^{2 \pi i \theta}\right)=1$ and $h(n)\left(e^{2 \pi i(\theta-n \alpha)}\right)=0$. Letting $f(0)=g(0)=$ $\frac{1}{2} k(0)$ and defining $f, g$ in $C_{c}(\mathbf{Z}, C(\mathbf{T}))$ in the obvious way, we have $f+g=k$, with $f \in K \cap N\left(\varphi_{\theta}\right)^{*}$ and $g \in K \cap N\left(\varphi_{\theta}\right)$. Thus the inclusion $(*)$ is true.

Given $\theta, \theta^{\prime}$ in $[0,1]$ with $e^{2 \pi i \theta^{\prime}}=e^{2 \pi i(\theta+n \alpha)}$, for some $n$ in $\mathbf{Z}$, then $\varphi_{\theta} \circ$ ad $U^{n}=\varphi_{\theta^{\prime}}$ on $A_{\alpha}$ (as they agree on the dense subalgebra generated by $U$ and $V$ ).

Proposition 4.2. For $\theta, \theta^{\prime}$ in $[0,1], \| \varphi_{\theta} \circ$ ad $x-\varphi_{\theta^{\prime}} \| \geq 1$ for all $x$ in $A_{\alpha}$ iff $e^{2 \pi i \theta^{\prime}} \notin\left\{e^{2 \pi i(\theta+n \alpha)} \mid n \in \mathbf{Z}\right\}$.

Proof. If $e^{2 \pi i \theta^{\prime}}=e^{2 \pi i(\theta+n \alpha)}$ for some $n$ in $\mathbf{Z}$, we have seen that there is an $x$, namely $U^{n}$, with $\varphi_{\theta} \circ$ ad $x=\varphi_{\theta^{\prime}}$.

Now suppose $e^{2 \pi i \theta^{\prime}} \notin\left\{e^{2 \pi i(\theta+n \alpha)} \mid n \in \mathbf{Z}\right\}$. For fixed $x$ in $C_{c}(\mathbf{Z}, C(\mathbf{T}))$ we find $b$ in $C(\mathbf{T})$ with $\varphi_{\theta} \circ$ ad $x(b)=0$ and $\varphi_{\theta^{\prime}}(b)=1$. As $x$ is in $C_{c}(\mathbf{Z}, C(\mathbf{T}))$, there is an $m$ in $\mathbf{Z}$ with support $x \subseteq\{-m, \ldots, m\}$. The hypothesis on $\theta, \theta^{\prime}$ allows us to find $b$ in $C(\mathbf{T})$ with $b \geq 0,\|b\|=1$, $b\left(e^{2 \pi i \theta^{\prime}}\right)=1$ and $b\left(e^{2 \pi i(\theta+n \alpha)}\right)=0$ for $n \in\{-m, \ldots, m\}$. Then

$$
\begin{aligned}
\varphi_{\theta} \circ \text { ad } x(b) & =\varphi_{\theta}\left(x * b * x^{*}(0)\right) \\
& =\sum_{n \in\{-m, \ldots, m\}} x(n) \alpha_{n}(b) \overline{x(n)}\left(e^{2 \pi i \theta}\right)=0
\end{aligned}
$$

(as $\alpha_{n}(b)\left(e^{2 \pi i \theta}\right)=0$ for $\left.n \in\{-m, \ldots, m\}\right)$, while $\varphi_{\theta^{\prime}}(b)=b\left(e^{2 \pi i \theta^{\prime}}\right)=1$. Thus $\| \varphi_{\theta} \circ$ ad $x-\varphi_{\theta^{\prime}} \| \geq 1$ for $x$ in $C_{c}(\mathbf{Z}, C(\mathbf{T}))$. With $x$ in $A_{\alpha}$ we choose a sequence $x_{j}$ in $C_{c}(\mathbf{Z}, C(\mathbf{T}))$ converging to $x$ in norm. Thus $\lim _{j \rightarrow \infty} \| \varphi_{\theta} \circ$ ad $x_{j}-\varphi_{\theta} \circ$ ad $x \|=0$ and the proposition follows.

If a state $\varphi$ is unitarily equivalent to a state $\psi$, that is, if $\varphi \circ$ ad $W=\psi$ for some unitary $W$ in $A_{\alpha}$, we write $\varphi \sim \psi$.

CoRollaRy 4.3. For $\theta, \theta^{\prime}$ in $[0,1], \varphi_{\theta} \sim \varphi_{\theta^{\prime}}$ iff $e^{2 \pi i \theta^{\prime}} \in\left\{e^{2 \pi i(\theta+n \alpha)} \mid\right.$ $n \in \mathbf{Z}\}$. 
As $\beta_{g}$ (for $g$ in $\operatorname{SL}(2, \mathbf{Z})$ ) is a ${ }^{*}$-automorphism of $A_{\alpha}$, the states $\varphi_{\theta} \circ \beta_{g}$ are pure. We examine what these states look like on the dense subalgebra $\left\{\Sigma_{\text {finite }} a_{p n} V^{p} U^{n} \mid a_{p n} \in \mathbf{C}\right\}$ of $A_{\alpha}$. Recall

$$
\beta_{g}\left(V^{p} U^{n}\right)=\xi(p, n)^{-1} \xi(\tilde{p}, \tilde{n}) V^{\tilde{p}} U^{\tilde{n}},
$$

where $(\tilde{p}, \tilde{n})=g(p, n)$ and $\xi(p, n)=e^{\pi i \alpha p n}(p, n$ in $\mathbf{Z})$. Thus

$$
\beta_{g}\left(\sum a_{p n} V^{p} U^{n}\right)=\sum a_{p n} \xi(p, n)^{-1} \xi(\tilde{p}, \tilde{n}) V^{\tilde{p}} U^{\tilde{n}}=\sum c_{\tilde{p} \tilde{n}} V^{\tilde{p}} U^{\tilde{n}},
$$

where

$$
c_{\tilde{p}, \tilde{n}}=a_{g^{-1}(\tilde{p}, \tilde{n})} \xi\left(g^{-1}(\tilde{p}, \tilde{n})\right)^{-1} \xi(\tilde{p}, \tilde{n})
$$

We have

$$
E\left(\sum a_{p n} V^{p} U^{n}\right)=\sum a_{p, 0} V^{p}
$$

so

$$
E\left(\beta_{g}\left(\sum a_{p n} V^{p} U^{n}\right)\right)=E\left(\sum c_{\tilde{p} \tilde{n}} V^{\tilde{p}} U^{\tilde{n}}\right)=\sum c_{\tilde{p}, \tilde{0}} V^{\tilde{p}}
$$

Thus

$$
\varphi_{\theta}\left(\beta_{g}\left(\sum a_{p n} V^{p} U^{n}\right)\right)=\sum_{\tilde{p}} c_{\tilde{p}, 0} e^{2 \pi i \tilde{p} \theta}
$$

For $g=\left[\begin{array}{l}h r \\ b\end{array}\right]$ in $\operatorname{SL}(2, \mathbf{Z})$,

$$
g^{-1}=\left[\begin{array}{cc}
d & -r \\
-b & h
\end{array}\right] \quad \text { and } \quad c_{\tilde{p}, 0}=a_{(d \tilde{p},-b \tilde{p})} \xi(d \tilde{p},-b \tilde{p})^{-1}
$$

Thus

$$
\varphi_{\theta}\left(\beta_{g}\left(\sum a_{p n} V^{p} U^{n}\right)\right)=\sum_{k} a_{(d k,-b k)} \xi(d k,-b k)^{-1} e^{2 \pi i k \theta} .
$$

Note that if $g$ is in the subgroup $H=\left\{\left[\begin{array}{ll}1 & n \\ 0 & 1\end{array}\right] \mid n \in \mathbf{Z}\right\}$ of $\operatorname{SL}(2, \mathbf{Z})$, then $\varphi_{\theta} \circ \beta_{g}=\varphi_{\theta}$ on $\left\{\sum_{\text {finite }} a_{p n} V^{p} U^{n} \mid a_{p n} \in \mathrm{C}\right\}$, so $\varphi_{\theta} \circ \beta_{g}=\varphi_{\theta}$ on $A_{\alpha}$. Also if $g$ is of the form $\left[\begin{array}{cc}-1 & n \\ 0 & -1\end{array}\right]$ for some $n$ in $\mathbf{Z}$ (so $-g \in H$ ), we have $\varphi_{\theta} \circ \beta_{g}=\varphi_{1-\theta}$.

Proposition 4.4. If $\theta, \theta^{\prime} \in[0,1], g \in S L(2, \mathbf{Z})$ and $\varphi_{\theta^{\prime}} \circ \beta_{g}$ is unitarily equivalent to $\varphi_{\theta}$, then $g$ or $-g$ is in $H=\left\{\left[\begin{array}{ll}1 & n \\ 0 & 1\end{array}\right] \mid n \in \mathbf{Z}\right\}$.

Proof. Choose $\left\{B_{n}\right\}$ a sequence in $C_{c}(\mathbf{Z}, C(\mathbf{T}))$ with $B_{n} \rightarrow W$ (in norm), where $W$ is a unitary in $A_{\alpha}$ with $\varphi_{\theta^{\prime}} \circ \beta_{g}=\varphi_{\theta} \circ$ ad $W$. Thus

$$
\sum_{m}\left|B_{n}(m)\right|^{2}=E\left(B_{n} B_{n}^{*}\right) \rightarrow E\left(W W^{*}\right)=E(\mathrm{Id})=\operatorname{Id}_{C(\mathbf{T})}
$$


in the norm of $C(\mathbf{T})$ as $n \rightarrow \infty$. Let $\varepsilon>0$ be given. Choose $n_{0}$ in $\mathbf{N}$ with $\left\|E\left(B_{n} B_{n}^{*}\right)-\operatorname{Id}_{C(\mathbf{T})}\right\|<\varepsilon / 4$ and $\| \varphi_{\theta} \circ$ ad $W-\varphi_{\theta} \circ$ ad $B_{n} \|<\varepsilon / 4$ for $n \geq$ $n_{0}$. As support $B_{n_{0}}$ is finite and $\alpha$ is irrational, we can choose $p$ in $\mathbf{Z} \backslash\{0\}$ with $\left|e^{2 \pi i p \alpha m}-1\right|<\varepsilon / 4$ for all $m$ in support $B_{n_{0}}$. Thus

$$
\varphi_{\theta}\left(B_{n_{0}} V^{p} B_{n_{0}}^{*}\right)=\rho_{\theta}\left(E\left(B_{n_{0}} V^{p} B_{n_{0}}^{*}\right)\right)=\sum_{m} e^{2 \pi i p(\theta+m \alpha)}\left(\left|B_{n_{0}}(m)\right|^{2}\left(e^{2 \pi i \theta}\right)\right)
$$

is within $\varepsilon / 4(1+\varepsilon / 4)$ of $e^{2 \pi i p \theta} E\left(B_{n_{0}} B_{n_{0}}^{*}\right)\left(e^{2 \pi i \theta}\right)$, which is within $\varepsilon / 4$ of $e^{2 \pi i p \theta}$. Thus $\varphi_{\theta}$ ad $W\left(V^{p}\right)$ is within $\varepsilon$ of $e^{2 \pi i p \theta}$. However,

$$
\varphi_{\theta^{\prime}} \beta_{g}\left(V^{k}\right)=\left\{\begin{array}{lll}
0 & \text { if } & b k \neq 0 \\
e^{2 \pi i h k \theta^{\prime}} & \text { if } & b k=0
\end{array}\right.
$$

for $k$ in $\mathbf{Z}$ and $g=\left[\begin{array}{l}h r \\ b d\end{array}\right]$ in $\operatorname{SL}(2, \mathbf{Z})$. Choosing $\varepsilon$ small enough and letting $k=p$ (so $k$ is non-zero), we have $\varphi_{\theta}, \beta_{g}\left(V^{p}\right)=\varphi_{\theta} \circ$ ad $W\left(V^{p}\right)$ only if $b=0$. Thus $g$ or $-g$ is in $H$.

Corollary 4.5. For $\theta, \boldsymbol{\theta}^{\prime}$ in $[0,1], g$ in $\operatorname{SL}(2, \mathbf{Z})$, we have $\varphi_{\theta^{\prime}} \circ \beta_{g}$ unitarly equivalent to $\varphi_{\theta}$ iff $g \in H$ and $e^{2 \pi i \theta^{\prime}} \in\left\{e^{2 \pi i(\theta+n \alpha)} \mid n \in \mathbf{Z}\right\}$ or $-g \in H$ and $e^{-2 \pi i \theta^{\prime}} \in\left\{e^{2 \pi i(\theta+n \alpha)} \mid n \in \mathbf{Z}\right\}$.

Proof. If $\varphi_{\theta^{\prime}} \circ \beta_{g} \sim \varphi_{\theta}$, we have $g$ or $-g$ in $H$. If $g \in H, \varphi_{\theta^{\prime}} \circ \beta_{g}=\varphi_{\theta^{\prime}}$, so $\varphi_{\theta^{\prime}} \sim \varphi_{\theta}$. We know this is equivalent to $e^{2 \pi i \theta^{\prime}} \in\left\{e^{2 \pi i(\theta+n \alpha)} \mid n \in \mathbf{Z}\right\}$. If $-g \in H$, then $\varphi_{\theta^{\prime}} \circ \beta_{g}=\varphi_{1-\theta^{\prime}}$, so $\varphi_{1-\theta^{\prime}} \sim \varphi_{\theta}$, which is equivalent to $e^{-2 \pi i \theta^{\prime}}$ $\in\left\{e^{2 \pi i(\theta+n \alpha)} \mid n \in \mathbf{Z}\right\}$. The reverse implication of the corollary is immediate.

We now show that the set of pure states $\left\{\varphi_{\theta} \circ \beta_{g} \mid \theta \in[0,1], g \in\right.$ $\mathrm{SL}(2, \mathbf{Z})\}$ separate the points of $A_{\alpha}$, i.e., if $x$ in $A_{\alpha}$ is non-zero, then $\varphi_{\theta} \circ \beta_{g}(x) \neq 0$ for some $\theta$ and $g$.

We proceed with some preliminary calculations. For $x=\sum_{\text {finite }} a_{r s} V^{r} U^{s}$ in $A_{\alpha}, \tau$ the trace on $A_{\alpha}$, and $m, n$ in $\mathbf{Z}$, we have

$$
\left|\tau\left(x V^{-m} U^{-n}\right)\right|=\left|\tau\left(\sum a_{r s} V^{r-m} U^{s-n}\right)\right|=\left|a_{m n}\right| .
$$

If $s=$ g.c.d. $(m, n)$, choose $g=\left[\begin{array}{l}h r \\ b d\end{array}\right]$ in $\operatorname{SL}(2, \mathbf{Z})$ with $g(m, n)=(s, 0)$. We have $g^{-1}=\left[\begin{array}{cc}d & -r \\ -b & h\end{array}\right]$ and $g^{-1}(s, 0)=(m, n)$, so $d s=m$ and $-b s=n$. We saw (in computing $\varphi_{\theta}\left(\beta_{g}(x)\right)$ that

$$
E\left(\beta_{g}(x)\right)=E\left(\beta_{g}\left(\sum a_{r s} V^{r} U^{s}\right)\right)=\sum_{k} a_{(d k,-b k)} \xi(d k,-b k)^{-1} V^{k},
$$


so

$$
\left|\tau\left(E\left(\beta_{g}(x)\right) V^{-s}\right)\right|=\left|a_{(d s,-b s)}\right|=\left|a_{(m, n)}\right| .
$$

The algebra $\left\{\sum_{\text {finite }} a_{r s} V^{r} U^{s} \mid a_{r s} \in \mathbf{C}\right\}$ is dense in $A_{\alpha}$ so continuity yields

$$
\left|\tau\left(x V^{-m} U^{-n}\right)\right|=\left|\tau\left(E\left(\beta_{g}(x)\right) V^{-s}\right)\right|
$$

for $x$ in $A_{\alpha}$.

Proposition 4.6. The set of pure states $\left\{\varphi_{\theta} \beta_{g} \mid \theta \in[0,1], g \in \operatorname{SL}(2, Z)\right\}$ separate the elements of $A_{\alpha}$.

Proof. If $\delta_{(r, j)}$ denotes the element of $L^{2}(\mathbf{Z} \times \mathbf{Z})$ which is one at $(r, j)$, zero elsewhere, we define unitaries $\pi_{\tau}(U), \pi_{\tau}(V)$ on $L^{2}(\mathbf{Z} \times \mathbf{Z})$ by

$$
\begin{gathered}
\pi_{\tau}(V): \delta_{(r, j)} \rightarrow \delta_{(r+1, j)}, \\
\pi_{\tau}(U): \delta_{(r, j)} \rightarrow e^{2 \pi i r \alpha} \delta_{(r, j+1)} .
\end{gathered}
$$

As $\pi_{\tau}(U) \pi_{\tau}(V)=e^{2 \pi i \alpha} \pi_{\tau}(V) \pi_{\tau}(U), A_{\alpha}$ is isomorphic to the $C^{*}$-algebra generated by $\pi_{\tau}(V), \pi_{\tau}(U)$.

Denoting this isomorphism by $\pi_{\tau}$ we note that $\pi_{\tau}$ with cyclic vector $\delta_{(0,0)}$ is the GNS representation of $A_{\alpha}$ associated with the trace $\tau$. For $x$ non-zero in $A_{\alpha}, \pi_{\tau}(x) \neq 0$, and we have $p, q, r, j$ in $\mathbf{Z}$ with $\left\langle\pi_{\tau}(x) \delta_{(p, q)}, \delta_{(r, j)}\right\rangle \neq 0$. Now

$$
\begin{aligned}
\left|\left\langle\pi_{\tau}(x) \pi_{\tau}\left(V^{p} U^{q}\right) \delta_{(0,0)}, \pi_{\tau}\left(V^{r} U^{j}\right) \delta_{(0,0)}\right\rangle\right| \\
=\left|\left\langle\pi_{\tau}\left(U^{-j} V^{-r} x V^{p} U^{q}\right) \delta_{(0,0)}, \delta_{(0,0)}\right\rangle\right| \\
=\left|\tau\left(U^{-j} V^{-r} x V^{p} U^{q}\right)\right|=\left|\tau\left(x V^{p} U^{q} U^{-j} V^{-r}\right)\right|=\left|\tau\left(x V^{p-r} U^{q-j}\right)\right|,
\end{aligned}
$$

so there are $m, n$ in $\mathbf{Z}$ with $\left|\tau\left(x V^{-m} U^{-n}\right)\right| \neq 0$. The preliminary calculation shows there is a $g$ in $\operatorname{SL}(2, \mathbf{Z}), s$ in $\mathbf{N}$ with $\tau\left(E\left(\beta_{g}(x)\right) V^{-s}\right) \neq 0$. Thus $E \beta_{g}(x) V^{-s} \neq 0$ and $E \beta_{g}(x) \neq 0$. For some $\theta$ in $[0,1], 0 \neq \rho_{\theta}\left(E\left(\beta_{g}(x)\right)\right)=$ $\varphi_{\theta}\left(\beta_{g}(x)\right)$.

5. Irreducible representations of $A_{\alpha}$ and ergodic actions. Given a factor representation $\pi$ of $A_{\alpha}$, we associated with it a cardinal $m$, namely the multiplicity of the representation restricted to the $C^{*}$-algebra generated by $V$. We can, of course, associate a second cardinal $m^{\prime}$ (and a second ergodic quasi-invariant regular Borel measure $\nu^{\prime}$ on $\mathbf{T}$ ) with $\pi$, where the restriction of $\pi$ to the $C^{*}$-algebra generated by $U$ (which is 
isomorphic to $C(\mathbf{T}))$ is unitarily equivalent via a unitary $W: \oplus^{m} L^{2}(\mathbf{T}, \nu)$ $\rightarrow \oplus^{m^{\prime}} L^{2}\left(\mathbf{T}, \nu^{\prime}\right)$ to the representation $g \rightarrow \oplus^{m^{\prime}} M_{g}$ of $C(\mathbf{T})$ of uniform multiplicity $m^{\prime}$ on $\oplus^{m^{\prime}} L^{2}\left(\mathbf{T}, \nu^{\prime}\right)$. If $(m, \nu, b)$ is the triple associated with $\pi$ and $f_{n}$ the function $t \rightarrow t^{n}$ on $\mathbf{T}$, then $b_{n}=W^{*} \oplus^{m} M_{f_{n}} W \tilde{U}_{n}^{-1}(n \in \mathbf{Z})$ (as $\left.\pi\left(U^{n}\right)=b_{n} \tilde{U}_{n}\right)$

In the proposition that follows, we describe a sufficient condition for factor representations of $A_{\alpha}$ to be irreducible.

PROPOSITION 5.1. If $\pi$ is a factor representation of $A_{\alpha}$ and the restriction of $\pi$ to the abelian $C^{*}$-subalgebras generated by $V$ and $U$ are of uniform finite multiplicities $m$ and $m^{\prime}$, respectively, and $m$ and $m^{\prime}$ are relatively prime, then $\pi$ is an irreducible representation.

Proof. Let $A_{1}$ and $A_{2}$ be the abelian $C^{*}$-subalgebras of $A_{\alpha}$ generated by $V$ and $U$, respectively. By assumption, $\pi\left(A_{1}\right)^{\prime}$ and $\pi\left(A_{2}\right)^{\prime}$ are von Neumann algebras by types $\mathrm{I}_{m}$ and $\mathrm{I}_{m^{\prime}}$, respectively. Each contains $\pi\left(A_{\alpha}\right)^{\prime}$, and $\pi\left(A_{\alpha}\right)^{\prime}$ is a factor by assumption. From Proposition 2.2., as $m$ (or $\left.m^{\prime}\right) \in \mathbf{N}, \pi\left(A_{\alpha}\right)^{\prime}$ is type I. Suppose $\pi\left(A_{\alpha}\right)^{\prime}$ is of type $\mathrm{I}_{n}$. Then there are $n$ orthogonal equivalent projections $E_{1}, \ldots, E_{n}$ in $\pi\left(A_{\alpha}\right)^{\prime}$ with sum I. This equivalence persists in $\pi\left(A_{1}\right)^{\prime}$. Since $\sum E_{k}=\mathrm{I}$, each $E_{k}$ has central carrier I in $\pi\left(A_{1}\right)^{\prime}$. From [10] there is a non-zero central projection $Q$ in $\pi\left(A_{1}\right)^{\prime}$ such that $Q E_{1}$ is the sum of $j$ equivalent abelian projections in $\pi\left(A_{1}\right)^{\prime}$. Since $Q E_{1}$ and $Q E_{k}$ are equivalent in $\pi\left(A_{1}\right)^{\prime}, Q E_{k}$ is the sum of $j$ equivalent abelian projections in $\pi\left(A_{1}\right)^{\prime}$ for each $k$ in $\{1, \ldots, n\}$. But $Q=\Sigma Q E_{k}$, so $Q$ is the sum of $n j$ equivalent abelian projections in $\pi\left(A_{1}\right)^{\prime}$. Now $\pi\left(A_{1}\right)^{\prime} Q$ is of type $\mathrm{I}_{m}$, so $m=n j$ and $n$ divides $m$. This same argument applied to $\pi\left(A_{2}\right)^{\prime}$ and $m^{\prime}$ yields the fact that $n$ divides $m^{\prime}$. By hypothesis, $m$ and $m^{\prime}$ are relatively prime. Thus $n=1 ; \pi\left(A_{\alpha}\right)^{\prime}$ is a factor of type $\mathrm{I}_{1}$, so $\pi\left(A_{\alpha}\right)^{\prime}$ consists of scalars and $\pi$ is irreducible.

We shall see examples of irreducible representations of $A_{\alpha}$ for which the finite multiplicities $m$ and $m^{\prime}$ above are any given pair of non-zero relatively prime numbers.

Let $\left(\rho_{m}, T\right)$ be the covariant representation associated to a factor representation $\pi$ of $A_{\alpha}$. Thus $\rho_{m}$ is a representation of $C(\mathbf{T})$ with uniform multiplicity $m \in \mathbf{N} \cup\{\infty\}$ on $\bigoplus^{m} L^{2}(\mathbf{T}, \nu)$ with $\nu$ a quasi-invariant ergodic regular Borel measure on $\mathbf{T}$ and $T_{n}=h_{n} \tilde{U}_{n}$, where $h_{n}$ is a unitary cocycle in $\mathfrak{M}_{m}\left(L^{\infty}(\mathbf{T}, \nu)\right)$.

Proposition 5.2. Let $\pi,\left(\pi_{m}, T\right)$ be as above. The representation $\pi$ is irreducible iff ad $T$ is an ergodic automorphism on $\mathfrak{M}_{m}\left(L^{\infty}(\mathbf{T}, \nu)\right)$. 
Proof. Note $C \in \pi\left(A_{\alpha}\right)^{\prime}$ iff $C \in \rho_{m}(C(\mathbf{T}))^{\prime}=\mathfrak{M}_{m}\left(L^{\infty}(\mathbf{T}, \nu)\right)$ and $T C T^{*}=C$. Thus $\pi\left(A_{\alpha}\right)^{\prime}=\mathbf{C}$ Id iff for $C$ in $\mathfrak{M}_{m}\left(L^{\infty}(\mathbf{T}, \nu)\right)$, ad $T(C)=C$ implies $C \in \mathbf{C}$ Id.

Note that if $m=1$, then ad $T$ is the ergodic automorphism $\alpha$ on $L^{\infty}(\mathbf{T}, \nu)$, so $\pi$ is an irreducible representation. In the next paragraphs, explicit irreducible representations of $A_{\alpha}$ are given for all $m$ in $\mathbf{N}$. These examples also serve to show that there are irreducible representations of $A_{\alpha}$ for which any pair of non-zero relatively prime numbers $m$ and $m^{\prime}$ can occur as the multiplicities (obtained by restricting the representation to the $C^{*}$-algebras generated by $V$ and $U$ respectively). Choose $b, d$ in $\mathbf{N} \backslash\{0\}$ with g.c.d. $(b, d)=1$. There are $z, k$ in $\mathbf{Z}$ such that $g=\left[\begin{array}{c}z k \\ b d\end{array}\right] \in \operatorname{SL}(2, \mathbf{Z})$. For $\theta \in[0,1]$ the (necessarily) irreducible representation associated with the pure state $\varphi_{\theta} \circ \beta_{g}$ is a representation of $A_{\alpha}$ whose restriction to $C(\mathbf{T})$ has multiplicity $b$. It will also be clear that the uniform multiplicity of this irreducible representation restricted to the $C^{*}$-algebra generated by $U$ is $d$. Note that given $b, d$ in $\mathbf{N} \backslash\{0\}$, there are many elements $g=\left[\begin{array}{c}z d \\ b d\end{array}\right]$ in $\operatorname{SL}(2, \mathbf{Z})$, however, given two such $g_{1}=\left[\begin{array}{ll}z & k \\ b & d\end{array}\right]$ and $g_{2}=\left[\begin{array}{ll}z^{\prime} & k^{\prime} \\ b & d\end{array}\right]$, we have

$$
g_{2} g_{1}^{-1} \in H=\left\{\left[\begin{array}{cc}
1 & n \\
0 & 1
\end{array}\right] \mid n \in \mathbf{Z}\right\}
$$

Thus $\varphi_{\theta} \circ \beta_{g_{1}}$ is equivalent to $\varphi_{\theta} \circ \beta_{g_{2}}$.

We first find a representation $\tilde{\pi}$ of $A_{\alpha}$ associated with the pure state $\varphi_{\theta} \circ \beta_{g}$. Define $\delta$ in $L^{2}(\mathbf{Z})$ by

$$
\delta_{n}(m)= \begin{cases}0 & \text { if } m \neq n \\ 1 & \text { if } m=n\end{cases}
$$

Define unitaries $\tilde{\pi}(V), \tilde{\pi}(U)$ on $L^{2}(\mathbf{Z})$ by describing their effect on the basis $\left\{\delta_{n} \mid n \in \mathbf{Z}\right\}$ of $L^{2}(\mathbf{Z})$.

$$
\begin{gathered}
\tilde{\pi}(V): \delta_{r} \rightarrow e^{2 \pi l d^{-1} \theta} e^{-2 \pi i d^{-1} r \alpha} \delta_{r+b}, \\
\tilde{\pi}(U): \delta_{r} \rightarrow e^{\pi i \alpha} \delta_{r+d} .
\end{gathered}
$$

We have $\tilde{\pi}(U) \tilde{\pi}(V)=e^{2 \pi i \alpha} \tilde{\pi}(V) \tilde{\pi}(U)$. Thus $A_{\alpha}$ is *-isomorphic to the $C^{*}$-algebra generated by $\tilde{\pi}(V), \tilde{\pi}(U)$, the ${ }^{*}$-isomorphism (denoted $\tilde{\pi}$ ) mapping $V$ to $\tilde{\pi}(V)$ and $U$ to $\tilde{\pi}(U)$.

If $p, n \in \mathbf{Z}$ then

$$
\begin{aligned}
\tilde{\pi}\left(V^{p} U^{n}\right): \delta_{r} \rightarrow & e^{\pi i n \alpha} e^{2 \pi i p d^{-1} \theta} \\
& \times e^{-2 \pi i d^{-1} \alpha[p(r+n d)+p(p-1) b / 2]} \delta_{r+n d+p b},
\end{aligned}
$$


so

$$
\left\langle\tilde{\pi}\left(V^{p} U^{n}\right) \delta_{0}, \delta_{0}\right\rangle= \begin{cases}e^{2 \pi l p d^{-1} \theta} e^{-\pi i p n \alpha} & \text { if } n d+p b=0, \\ 0 & \text { otherwise, }\end{cases}
$$

which is equal to $\varphi_{\theta} \circ \beta_{g}\left(V^{p} U^{n}\right)$. As g.c.d. $(b, d)=1$, we can choose $p, n$ such that $n d+p b$ is any preassigned integer. Thus $\delta_{0}$ is a cyclic vector for the representation $\tilde{\pi}$ of $A_{\alpha}$. Linearity and continuity imply $\left\langle\tilde{\pi}(x) \delta_{0}, \delta_{0}\right\rangle=$ $\varphi_{\theta} \circ \beta_{g}(x)$ for all $x \in A_{\alpha}$. The representation $\tilde{\pi}$ of $A_{\alpha}$ is associated with the pure state $\varphi_{\theta} \circ \beta_{g}$, so it is irreducible.

We show that $\left.\tilde{\pi}\right|_{C(\mathbf{T})}$ is unitarily equivalent to the multiplicity $b$ representation $\rho_{b}$ with Haar measure on $\mathbf{T}$. Investigating $\left.\tilde{\pi}\right|_{C(\mathbf{T})}$, where $C(\mathbf{T})$ is the $C^{*}$-algebra generated by $V$, we see that $L^{2}(\mathbf{Z})$ is the direct sum of $b$ subspaces $\left\{H_{j}\right\}_{j}^{b-1}=0$, where $H_{J}$ is the $\left.\tilde{\pi}\right|_{C(\mathbf{T})}$ invariant subspace generated by $\left\{\delta_{q b+j} \mid q \in \mathbf{Z}\right\}, j=0, \ldots, b-1$. If $W$, is the partial isometry on $L^{2}(\mathbf{Z})$ with initial space $H$, and final space $H_{0}$ determined by

$$
W_{J}: \delta_{q b+j} \rightarrow e^{2 \pi l d^{-1} \alpha q j} \delta_{q b} \quad(q \in \mathbf{Z}),
$$

then $W=\bigoplus_{j=0}^{b-1} W_{J}$ is a unitary transformation from $\bigoplus_{0}^{b-1} H_{J}$ onto $\bigoplus_{0}^{b-1} H_{0}$, with $W\left(\left.\tilde{\pi}\right|_{C(\mathbf{T})}(x)\right)=\left(\oplus_{0}^{b-1} \pi(x)\right) W$ (as $W_{J} \tilde{\pi}(V) \delta_{q b+\jmath}=$ $\left.\tilde{\pi}(V) W \delta_{q b+\jmath}\right)$, where $\pi$ is $\left.\tilde{\pi}\right|_{C(\mathbf{T})}$ restricted to the subspace $H_{0}$ and $x \in C(\mathbf{T})$.

We show $\pi$ is equivalent to the multiplication representation of $C(\mathbf{T})$ on $L^{2}(\mathbf{T}, \mu)$ where $\mu$ is Haar measure on $\mathbf{T}$. If $f$ is an element of $B=\left\{\sum_{\text {finite }} a_{n} V^{n} \mid a_{n} \in \mathbf{C}\right\}$, then

$$
\left\|\pi(f) \delta_{0}\right\|^{2}=\left\langle\pi\left(f^{*} f\right) \delta_{0}, \delta_{0}\right\rangle=\sum_{\text {finite }}\left|a_{n}\right|^{2}=\int_{\mathbf{T}}|f|^{2} d \mu .
$$

The linear map $Y$ defined on $B$, a norm dense subspace of $L^{2}(\mathbf{T}, \mu)$, to $H_{0}$ by $f \rightarrow \pi(f) \delta_{0}$ is therefore an isometry onto a dense subspace of $H_{0}$. The map $Y$ extends to a unitary map (also called $Y$ ) of $L^{2}(\mathbf{T}, \mu)$ onto $H_{0}$. For $x$ in $B$,

$$
Y^{*} \pi(V) Y(x)=Y^{*} \pi(V) \pi(x) \delta_{0}=Y^{*} \pi(V x) \delta_{0}=V x,
$$

so continuity ensures that $Y^{*} \pi(V) Y$ is multiplication by $V$ on $L^{2}(\mathbf{T}, \mu)$. Thus $\pi$ is unitarily equivalent via the unitary $Y^{*}$ to the multiplication representation of $C(\mathbf{T})$ on $L^{2}(\mathbf{T}, \mu)$, and $\left.\tilde{\pi}\right|_{C(\mathbf{T})}$ is unitarily equivalent to the multiplicity $b$ representation $\rho_{b}$ on $\bigoplus_{0}^{b-1} L^{2}(\mathbf{T}, \mu)$ via the unitary $\left(\oplus_{0}^{b-1} Y^{*}\right) W$. In an analogous manner we note that $\tilde{\pi}$ restricted to the $C^{*}$-algebra $C^{*}(U)$ generated by $U$ is (unitarily equivalent to) a multiplicity $d$ representation on $\bigoplus_{0}^{d-1} L^{2}(\mathbf{T}, \mu)$. 
To determine the unitary cocycle $h_{1}$ for the representation $\tilde{\pi}$ of $A_{\alpha}$, we examine what $\tilde{\pi}(U)$ looks like on $\oplus_{0}^{b-1} L^{2}(\mathbf{T}, \mu)$, i.e., we compute $\left(\oplus_{0}^{b-1} Y^{*}\right) W \tilde{\pi}(U) W^{*}\left(\oplus_{0}^{b-1} Y\right)$.

There are $a, s$ in $\mathbf{N}$ with $0<s<b, d=a b+s$ and g.c.d. $(b, s)=$ g.c.d. $(b, d)=1$. The unitary $\tilde{\pi}(U)$ maps $H_{j}$ isometrically onto $H_{j+s}$ if $0 \leq j<b-s$ and onto $H_{j-(b-s)}$ if $b-s \leq j \leq b-1$. Note that $Y\left(\gamma(n) V^{n}\right)=\delta_{n b}$ with

$$
\gamma(n)=e^{-2 \pi i n d^{-1} \theta} e^{\pi i d^{-1} \alpha b(n(n-1))},
$$

so (for $x, y \in\{0, \ldots, b-1\})$

$$
\begin{aligned}
& Y^{*} W_{x} \tilde{\pi}(U) W_{y}^{*} Y\left(V^{n}\right) \\
& =\left\{\begin{array}{c}
\gamma(n+a) \overline{\gamma(n)} e^{\pi i \alpha} e^{2 \pi i d^{-1} \alpha[n s+a x]} V^{n+a} \\
\text { if } s \leq x \leq b-1 \text { and } x-y=s, \\
\gamma(n+a+1) \overline{\gamma(n)} e^{\pi i \alpha} e^{2 \pi i d^{-1} \alpha[n(s-b)+(a+1) x]} V^{n+a+1} \\
\text { if } 0 \leq x<s \text { and } y-x=b-s, \\
0 \quad \text { otherwise. }
\end{array}\right.
\end{aligned}
$$

Letting

$$
\begin{aligned}
& g(x)=\left\{\begin{array}{l}
e^{-2 \pi i d^{-1} \theta a} e^{\pi i d^{-1} \alpha\left[2 a x+s+b a^{2}\right]} V^{a} \quad \text { if } s \leq x \leq b-1, \\
e^{-2 \pi i d^{-1} \theta(a+1)} e^{\pi i d^{-1} \alpha\left[2(a+1) x+2 a b+s+b a^{2}\right]} V^{a+1}
\end{array}\right. \\
& \text { if } 0 \leq x<s \text {, }
\end{aligned}
$$

we have

$$
Y^{*} W_{x} \tilde{\pi}(U) W_{y}^{*} Y\left(V^{n}\right)=e^{2 \pi i n \alpha} g(x) V^{n}=g(x) \alpha\left(V^{n}\right),
$$

where $x-y=s$ if $s \leq x \leq b-1$ and $y-x=b-s$ if $0 \leq x<s$.

Thus the unitary cocycle $h_{1}$ in $\mathfrak{M}_{b}\left(L^{\infty}(\mathbf{T}, \mu)\right)$ associated with $\left(\oplus_{0}^{b-1} Y^{*}\right) W \tilde{\pi}(U) W^{*}\left(\oplus_{0}^{b-1} Y\right)$ is the following matrix on $\bigoplus_{0}^{b-1} L^{2}(\mathbf{T}, \mu)$ :

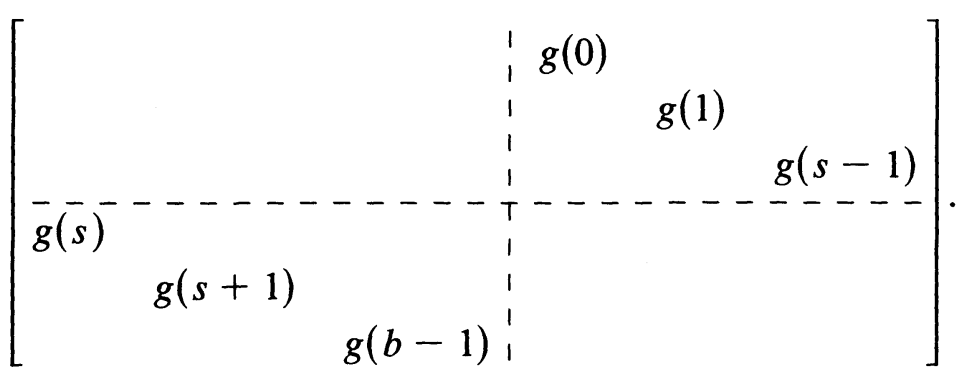


Noticing that the entries of this matrix are continuous functions on $\mathbf{T}$, we have the additional information that ad $T=\operatorname{ad} h_{I} \tilde{U}$ (see Proposition $5.2)$ is actually an ergodic automorphism of the $C^{*}$-algebra $\mathfrak{M}_{m}(C(\mathbf{T})$ ). We have thus found explicit examples of cocycles which perturb the non-ergodic action $\left[a_{l}\right] \rightarrow\left[\alpha\left(a_{l j}\right)\right]$ on $\mathfrak{M}_{m}(C(\mathbf{T}))(m>1)$ into an ergodic action.

We have inequivalent pure states with the same pair of relatively prime numbers as their multiplicities. For example, with $g=\left[\begin{array}{l}z k \\ b d\end{array}\right]$ as above, $\varphi_{\theta^{\prime}} \circ \beta_{g} \nsim \varphi_{\theta} \circ \beta_{g}$, with $\theta^{\prime}$ not in the orbit of $\theta$. Also if $g^{\prime}=\left[\begin{array}{cc}-z & k \\ b & -d\end{array}\right]$, then $g^{\prime} g^{-1} \notin H$, so $\varphi_{\theta^{\prime}} \circ \beta_{g} \nsim \varphi_{\theta} \circ \beta_{g^{\prime}}$ for all $\theta$ in $[0,1]$. One sees, however, that $\varphi_{\theta} \circ \beta_{g^{\prime}}$ has $b, d$ as its associated multiplicities (as does $\varphi_{\theta^{\prime}} \circ \beta_{g}$ ).

Remark added in proof. Y. Watatani has also considered a representation of $\mathrm{SL}(2, \mathbf{Z})$ as automorphisms of $A_{\alpha}$ in Toral automorphisms on irrational rotation algebras, Math. Japonica, 26, No. 4 (1981).

\section{REFERENCES}

[1] L. Baggett, Representations of the Mautner Group, I Pacific J. Math., 77 (1978).

[2] B. Brenken and R. Kadison to appear.

[3] A. Connes, A factor not antiisomorphic to itself, Annals of Math., 101 (1975), 536-554.

[4] J. Dixmier, Les $C^{*}$-algebras et Leurs Representations, Bordas, (Gauthier-Villars), Paris, 1969.

[5] S. Doplicher, D. Kastler and D. Robinson, Covariance algebras in field theory and statistical mechanics, Comm. Math. Phys., 3 (1966), 1-28.

[6] P. Green, The local structure of twisted covariance algebras, Acta Math., 140 (1978), 191-250.

[7] R. Herman and V. Jones, Period two automorphisms of U.H.F. C ${ }^{*}$-algebras, J. Functional Anal., 45 (1982), 169-176.

[8] R. Kadison, Representations of Matricial Operator Algebras, (to appear, Proc. Neptun Conf. on Op. Alg. and Group Rep. Pittman, London).

[9] _ Diagonalizing Matrices, (to appear).

[10] R. Kadison and J. Ringrose, Fundamentals of the Theory of Operator Algebras, Vols. I and II, Academic Press, New York (1982-83).

[11] G. Mackey, The theory of Unitary Group Representations, University of Chicago Press, 1976.

[12] G. Pedersen, $C^{*}$-Algebras and their Automorphism Groups, Academic Press, London, 1979.

[13] M. Pimsner and D. Voiculescu, Exact sequences for K-groups, J. Operator Theory, 4 No. 1 (1980), 93-118.

[14] __ Imbedding the irrational rotation $C^{*}$-algebra into an AF algebra, J. Operator Theory, 4 (1980).

[15] S. Popa and M. Reiffel, The Ext groups of the $C^{*}$-algebras associated with irrational rotations, J. Operator Theory, 3 No. 2 (1980).

[16] M. Reiffel, $C^{*}$-algebras associated with an irrational rotation, Pacific J. Math., 93 No. 2 (1981), 415-429. 
[17] S. Sakai, $C^{*}$-Algebras and $W^{*}$-Algebras, Springer Verlag, 1970.

[18] J. Taylor, Banach Algebras and Topology in "Algebras in Analysis", Academic Press, 1975, 118-186.

[19] V. Varadarajan, Geometry of Quantum Theory, Vol. 2, Van Nostrand 1970.

[20] G. Zeller-Meier, Produits Croises $d$ 'une $C^{*}$-algebra par un Groupes d'automorphismes, J. Math. Pures et Appl., 47 (1968), 101-239.

Received July 9, 1982 and in revised form November 30, 1982.

DALHOUSIE UNIVERSITY

HalifaX, Nova Scotia

CANADA, B3H-4H8 


\title{
PACIFIC JOURNAL OF MATHEMATICS EDITORS
}

\author{
Donald BABBITT (Managing Editor) \\ University of California \\ Los Angeles, CA 90024 \\ Hugo RossI \\ University of Utah \\ Salt Lake City, UT 84112 \\ C. C. MOore and Arthur Ogus \\ University of California \\ Berkeley, CA 94720
}

J. DUGUNDJI
Department of Mathematics

University of Southern California

Los Angeles, CA 90089-1113

R. FinN and H. SAMELSON

Stanford University

Stanford, CA 94305

\section{ASSOCIATE EDITORS}
R. ARENS
E. F. BECKENBACH
B. H. NeumanN
F. WOLF
K. YosHIDA (1906-1982)

\section{SUPPORTING INSTITUTIONS}

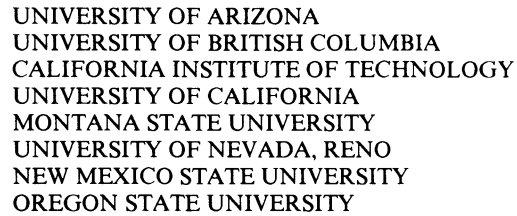

UNIVERSITY OF ARIZONA

UNIVERSITY OF BRITISH COLUMBIA

CALIFORNIA INSTITUTE OF TECHNOLOGY

UNIVERSITY OF CALIFORNIA

MONTANA STATE UNIVERSITY

UNIVERSITY OF NEVADA, RENO

NEW MEXICO STATE UNIVERSITY

OREGON STATE UNIVERSITY

\author{
UNIVERSITY OF OREGON \\ UNIVERSITY OF SOUTHERN CALIFORNIA \\ STANFORD UNIVERSITY \\ UNIVERSITY OF HAWAII \\ UNIVERSITY OF TOKYO \\ UNIVERSITY OF UTAH \\ WASHINGTON STATE UNIVERSITY \\ UNIVERSITY OF WASHINGTON
}

The Supporting Institutions listed above contribute to the cost of publication of this Journal, but they are not owners or publishers and have no responsibility for its content or policies.

Mathematical papers intended for publication in the Pacific Journal of Mathematics should be in typed form or offset-reproduced (not dittoed), double spaced with large margins. Please do not use built up fractions in the text of the manuscript. However, you may use them in the displayed equations. Underline Greek letters in red, German in green, and script in blue. The first paragraph must be capable of being used separately as a synopsis of the entire paper. In particular it should contain no bibliographic references. Please propose a heading for the odd numbered pages of less than 35 characters. Manuscripts, in triplicate, may be sent to any one of the editors. Please classify according to the scheme of Math. Reviews, Index to Vol. 39. Supply name and address of author to whom proofs should be sent. All other communications should be addressed to the managing editor, or Elaine Barth, University of California, Los Angeles, California 90024.

There are page-charges associated with articles appearing in the Pacific Journal of Mathematics. These charges are expected to be paid by the author's University, Government Agency or Company. If the author or authors do not have access to such Institutional support these charges are waived. Single authors will receive 50 free reprints; joint authors will receive a total of 100 free reprints. Additional copies may be obtained at cost in multiples of 50 .

The Pacific Journal of Mathematics is issued monthly as of January 1966. Regular subscription rate: $\$ 132.00$ a year (6 Vol., 12 issues). Special rate: $\$ 66.00$ a year to individual members of supporting institutions.

Subscriptions, orders for numbers issued in the last three calendar years, and changes of address should be sent to Pacific Journal of Mathematics, P.O. Box 969, Carmel Valley, CA 93924, U.S.A. Old back numbers obtainable from Kraus Periodicals Co., Route 100, Millwood, NY 10546.

The Pacific Journal of Mathematics ISSN 0030-8730 is published monthly by the Pacific Journal of Mathematics at P.O. Box 969, Carmel Valley, CA 93924. Application to mail at Second-class postage rates is pending at Carmel Valley, California, and additional mailing offices. Postmaster: Send address changes to Pacific Journal of Mathematics, P. O. Box 969, Carmel Valley, CA 93924.

PUBLISHED BY PACIFIC JOURNAL OF MATHEMATICS, A NON-PROFIT CORPORATION 


\section{Pacific Journal of Mathematics}

\section{Vol. 111, No. $2 \quad$ December, 1984}

Berndt Brenken, Representations and automorphisms of the irrational

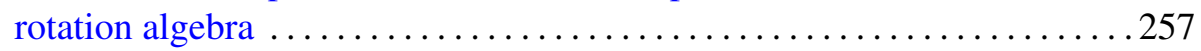

Harold George Diamond, A number theoretic series of I. Kasara . . . . . . 283

Rolf Farnsteiner, On the structure of simple-semiabelian Lie algebras . . . . 287

Guillermo Grabinsky, Poisson process over $\sigma$-finite Markov chains . ......301

Derbiau Frank Hsu and A. Donald Keedwell, Generalized complete

mappings, neofields, sequenceable groups and block designs. I . . . . . 317

William H. Julian and Fred Richman, A uniformly continuous function on

$[0,1]$ that is everywhere different from its infimum $\ldots \ldots \ldots \ldots . \ldots 33$

D. H. Lehmer and Emma Lehmer, The sextic period polynomial .........341

E. Maluta, Uniformly normal structure and related coefficients ...........357

Coy Lewis May, The species of bordered Klein surfaces with maximal

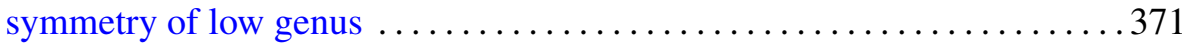

Louis Jackson Ratliff, Jr., On asymptotic prime divisors . . . . . . . . . . . 395

Norbert Riedel, Disintegration of KMS-states and reduction of standard von

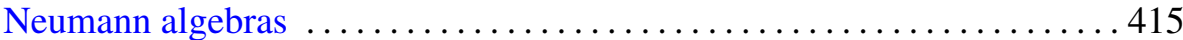

Richard Gordon Swan, $n$-generator ideals in Prüfer domains ...........433

Vilmos Totik, An interpolation theorem and its applications to positive

operators .................................. 447

Richard Vrem, Hypergroup joins and their dual objects 